\title{
Metric regularity and quantitative stability in stochastic programs with probabilistic constraints *
}

\author{
René Henrion and Werner Römisch \\ Humboldt-Universität zu Berlin \\ Institut für Mathematik \\ D-10099 Berlin, Germany
}

\begin{abstract}
Necessary and sufficient conditions for metric regularity of (several joint) probabilistic constraints are derived using recent results from nonsmooth analysis. The conditions apply to fairly general nonconvex, nonsmooth probabilistic constraints and extend earlier work in this direction. Further, a verifiable sufficient condition for quadratic growth of the objective function in a more specific convex stochastic program is indicated and applied in order to obtain a new result on quantitative stability of solution sets when the underlying probability distribution is subjected to perturbations. This is used to establish a large deviation estimate for solution sets when the probability measure is replaced by empirical ones.
\end{abstract}

\section{Keywords:}

stochastic programming, probabilistic constraints, metric regularity, nonsmooth analysis, quadratic growth, quantitative stability, large deviations

\section{AMS subject classifications:}

90C $15,90 \mathrm{C} 30,49 \mathrm{~J} 52$

${ }^{*}$ This research is supported by the Deutsche Forschungsgemeinschaft 


\section{Introduction}

When building stochastic models in decision making under (stochastic) uncertainty, the two main approaches consist in introducing future costs (e.g. for the compensation of constraint violations) and in fixing certain reliability levels for constraints. The latter approach is motivated by many problems in engineering sciences, where system reliability is an important feature (e.g. inventory control, power generation, structural design etc. [30], [38], [55]). It leads to stochastic programming problems with (so-called) probabilistic or chance constraints. To give a mathematical formulation of the model we study in this paper, let $\xi$ be an $s$-dimensional random vector on some probability space $(\Omega, \mathcal{A}, P)$ and let $\xi \in H_{j}(x), j=1, \ldots, d$, describe $d$ constraints depending on $\xi$ and on the decision vector $x \in \mathbb{R}^{m}$. Denoting by $g$ the objective function and by $C$ the subset of $\mathbb{R}^{m}$ expressing all deterministic constraints, we arrive at the following model:

$$
\min \left\{g(x) \mid x \in C, P\left(\xi \in H_{j}(x)\right) \geq p_{j}, j=1, \ldots, d\right\} .
$$

Here $p_{j} \in(0,1)$ denotes the probability (or reliability) level subject to which the constraint ' $\xi \in H_{j}(x)$ ' has to be satisfied. Since different reliability requirements might be fixed for different constraints, the levels $p_{j} \in(0,1), j=1, \ldots, d$ are allowed to be different. Later we shall prefer the following formulation of the model

$$
\mathbf{P}(\mu) \quad \min \left\{g(x) \mid x \in C, \mu\left(H_{j}(x)\right) \geq p_{j}, j=1, \ldots, d\right\},
$$

where $\mu$ denotes the probability distribution of $\xi$, i.e., $\mu=P \circ \xi^{-1}$. In Section 2 the assumptions on the data $g, C, H_{j}(j=1, \ldots, d)$ are specified, so that the model is well-defined and enjoys suitable properties. In Section 3 we shall study the case where $H_{j}$ has the form $H_{j}(x)=\{z \in$ $\left.\mathbb{R}^{s} \mid h_{j}(x) \geq z_{j}\right\}$ with $h_{j}: \mathbb{R}^{m} \rightarrow \mathbb{R}^{s_{j}}, j=1, \ldots, d$, and $\sum_{j=1}^{d} s_{j}=s$, and in Section 4 we shall deal with the polyhedral case, i.e., $C$ is polyhedral and $h=h_{1}$ is linear $(d=1)$.

In most practical applications of the stochastic programming methodology only incomplete information on the probability distribution $\mu$ (of $\xi$ ) is available. This fact and the possible need of approximations for $\mu$ in solution methods (cf. [31], [34], [38]) motivate a stability analysis of $\mathbf{P}(\mu)$ with respect to perturbations of $\mu$ in the space $\mathcal{P}\left(\mathbb{R}^{s}\right)$ of all Borel probability measures on $I R^{s}$ endowed with a suitable convergence (or metric). In the context of stochastic programs with probabilistic constraints, this problem was addressed in several papers, e.g. [1], [14], [15], [16], [22], [23], [24], [42], [43], [44], [46], [47], [48], [51], [52], [53], [54]. In [14] a nonlinear parametric framework is adapted to study stability with respect to changes of finite dimensional parameters of the distribution $\mu$. The convergence theory for measurable multifunctions is utilized in [47] to develop general approximation results for probabilistically constrained models. This approach is also used in [51], [52], leading to general, satisfactory results on convergence rates of estimates for such models. Further results in this direction are given in [23], [24]. Asymptotic properties of the optimal value based on an extended delta method are studied in [48]. Recently, a new class of nonparametric estimators that preserve convexity properties has been adapted to chance constrained models in [16]. The asymptotic

behaviour of these estimates and of solution sets to stochastic programs is analysed, too. In the remaining papers quoted above, stochastic programs are viewed as parametric programs 
with respect to the probability measure $\mu$. [22], [53] and [54] give qualitative stability results for constraint sets, marginal values and solutions when the measure $\mu$ is perturbed in $\mathcal{P}\left(\mathbb{R}^{s}\right)$ equipped with the (metrizable) topology of weak convergence ([6]). In [1], [42], [43], [44], [46] quantitative stability results for marginal values are obtained with respect to certain metric distances on $\mathcal{P}\left(\mathbb{R}^{s}\right)$ (the Prokhorov metric in [1] and so-called discrepancies in the other papers). The papers [42], [43], [44] also contain results on upper semicontinuity of local solution sets.

The aim of the present paper is to extend the results in [42], [43], [44] in two directions: earlier conditions on the stability of probabilistic constraint sets are considerably generalized and a novel result on the Hausdorff Hölder stability of solution sets is established. We start our analysis by stating a general quantitative stability result for $\mathbf{P}(\mu)$ (Theorem 2.1), which relies on the recent work by Klatte [26] and on techniques developed in [44], [46]. The crucial conditions in this result are the metric regularity of the probabilistic constraints and a quadratic growth condition for the objective function near non isolated minima. The growth condition appears in a more general context also in [2], [7], [49] for instance, and in a slightly different framework in [28]. The aim of our analysis is to derive verifiable conditions (on the original problem $\mathbf{P}(\mu)$ ) for metric regularity and quadratic growth. In particular, we focus on conditions that apply to nonsmooth probabilistic constraints in order to enlarge the range of applications. In Section 3 we obtain characterizations of metric regularity by exploiting the nonconvex subdifferential calculus by Mordukhovich ([32], [33]). Two types of sufficient conditions for metric regularity are developed. The first one represents an explicit growth condition for the composite function $\theta_{\mu}(x)=\left(\mu\left(H_{1}(x)\right), \ldots, \mu\left(H_{d}(x)\right)\right)$ at a feasible point (Theorem 3.12). The second type consists of separate constraint qualifications for the function $h=\left(h_{1}, \ldots, h_{d}\right)$ relative to $C$ and for a function $\Phi_{\mu}$ whose components are certain marginal distribution functions of $\mu$ (Theorem 3.16). In case $\mu$ has a density, a more transparent and verifiable condition, which implies the constraint qualification for $\Phi_{\mu}$, is established (Theorem 3.17). This can be achieved even globally if the strict positivity region of the density contains an infinity path (Theorem 3.21). The principal statements are illustrated by examples showing their validity and limitations. Earlier results are essentially extended (cf. the discussion following Proposition 2.2). In Section 4 we consider a particular convex stochastic program and give a criterion implying quadratic growth of the objective near the solution set. In this respect a local strong concavity property of the measure $\mu$ is essential. The methodology for proving this result (Theorem 4.2) is shown to extend to establishing the Hausdorff Hölder continuity for solution sets (Theorem 4.3). Finally, it is outlined that the latter result has immediate implications to rates of convergence for nonparametric estimation procedures in $\mathbf{P}(\mu)$. Namely, we derive a large deviation type result for the Hausdorff distance of solution sets if the original distribution $\mu$ is estimated by empirical measures.

\section{A general result on quantitative stability}

In this section, we develop a framework for stability analysis of probabilistic constrained models and present a general result on the quantitative stability of marginal values and (local) solution 
sets. We consider the stochastic programming model $\mathbf{P}(\mu)$ formulated in the introduction

$$
\mathbf{P}(\mu) \quad \min \left\{g(x) \mid x \in C, \mu\left(H_{j}(x)\right) \geq p_{j}, j=1, \ldots, d\right\},
$$

which involves several (joint) probabilistic constraints. For the data we assume that $g$ is a continuous mapping from $\mathbb{R}^{m}$ into $\mathbb{R}, C$ is a nonempty, closed subset of $\mathbb{R}^{m}, H_{j}$ is a set-valued mapping from $R^{m}$ into $\mathbb{R}^{s}$ having a closed graph (for each $j=1, \ldots, d$ ), $p_{j} \in$ $(0,1)(j=1, \ldots, d)$ and $\mu \in \mathcal{P}\left(\mathbb{R}^{s}\right)$. Making use of the notations $p=\left(p_{1}, \ldots, p_{d}\right)$ and $\left.M_{p}(\nu)=\left\{x \in C \mid \nu\left(H_{j}(x)\right) \geq p_{j}, j=1, \ldots, d\right)\right\}$ for each $\nu \in \mathcal{P}\left(\mathbb{R}^{s}\right)$, the model $\mathbf{P}(\mu)$ takes the form

$$
\min \left\{g(x) \mid x \in M_{p}(\mu)\right\} .
$$

The first step to analyse stability of (2) with respect to perturbations of $\mu$ in $\mathcal{P}\left(\mathbb{R}^{s}\right)$ is to identify a (suitable) metric distance on $\mathcal{P}\left(\mathbb{R}^{s}\right)$. Consistently with [46], [44] we consider the following distance, which is sometimes called $\mathcal{B}$-discrepancy:

$$
\alpha(\mu, \nu)=\sup \{|\mu(B)-\nu(B)| \mid B \in \mathcal{B}\}
$$

Here $\mathcal{B}$ is a class of closed subsets of $\mathbb{I R}^{s}$ such that all sets of the form $H_{j}(x)(x \in C ; j=$ $1, \ldots, d)$ belong to $\mathcal{B}$ and that $\mathcal{B}$ is a determining class (i.e., it has the property that if any two measures agree on $\mathcal{B}$, then they coincide). Convergence of a sequence of probability measures with respect to the metric $\alpha$ means its uniform convergence on $\mathcal{B}$. Necessary and sufficient conditions on $\mathcal{B}$ such that weak convergence of probability measures implies uniform convergence on $\mathcal{B}$ usually refer to certain uniformity properties of the class $\mathcal{B}$ with respect to the limit measure (cf. e.g. [4], [5]) or to the sequential compactness of $\mathcal{B}$, viewed as a subset of the hyperspace of closed subsets of $\mathbb{I R}^{s}$ equipped with a suitable topology ([29]). In particular, if $\mathcal{B}$ is a subclass of all convex Borel sets, then the uniform convergence on $\mathcal{B}$ to the limit measure $\mu$ is implied by its weak convergence and the condition $\mu(\partial B)=0$ for all $B \in \mathcal{B}$ ( $\partial B$ denoting the topological boundary of $B$ ).

A special feature of model (2) is that we have to take into account its possible nonconvexity. Even when the original model is convex (cf. e.g. Proposition 2.2), perturbations of $\mu$ (e.g. by discrete measures) lead to nonconvex perturbed programs. Hence, an appropriate concept for the stability analysis of (2) has to take into account the perturbation of sets of local minimizers. Here we make use of the concepts developed in [25], [39] and, in particular, of so-called complete minimizing sets (CLM sets). Given $V \subseteq \mathbb{R}^{m}$, we put for each $\nu \in \mathcal{P}\left(\mathbb{R}^{s}\right)$

$$
\varphi_{V}(\nu)=\inf \left\{g(x) \mid x \in M_{p}(\nu) \cap c l V\right\}
$$

and

$$
\Psi_{V}(\nu)=\operatorname{argmin}\left\{g(x) \mid x \in M_{p}(\nu) \cap c l V\right\}=\left\{x \in M_{p}(\nu) \cap c l V \mid g(x)=\varphi_{V}(\nu)\right\}
$$

where $c l V$ denotes the closure of $V$. Given $\mu \in \mathcal{P}\left(\mathbb{R}^{s}\right)$, we call a nonempty subset $X$ of $\mathbb{I}^{m}$ a CLM set for (2) with respect to $V$, if $V$ is an open subset of $\mathbb{R}^{m}$ containing $X$ and $X=\Psi_{V}(\mu)$. For a discussion of CLM sets we refer to [39], but mention that nonempty sets of 
global minimizers, isolated local minimizers and sets of non-isolated local minimizers around which $g$ satisfies a quadratic growth condition (cf. e.g. [7], [49], [26]) are examples of CLM sets.

To state our quantitative stability result, we still need a stability property for the probabilistic constraint in (2). We put $\theta_{\mu}: \mathbb{R}^{m} \rightarrow \mathbb{R}^{d}, \theta_{\mu}^{j}(x)=\mu\left(H_{j}(x)\right)$ for each $x \in \mathbb{R}^{m}, j=1, \ldots, d$, and $p=\left(p_{1}, \ldots, p_{d}\right) \in \mathbb{R}^{d}$. Consistently with the general definition given in Section 3 we say here that the probabilistic constraint function $\theta_{\mu}(\cdot)-p$ is metrically regular with respect to $C$ at some $x^{0} \in M_{p}(\mu)$ if there are constants $a>0$ and $\varepsilon>0$ such that

$$
\operatorname{dist}\left(x, M_{p-y}(\mu)\right) \leq a \cdot \operatorname{dist}\left(\theta_{\mu}(x)-p, \mathbb{R}_{+}^{d}-y\right)=a\left\|\max \left\{0, p-y-\theta_{\mu}(x)\right\}\right\|
$$

for all $(x, y) \in\left(C \cap B_{\varepsilon}\left(x^{0}\right)\right) \times B_{\varepsilon}(0)$. Here (and in all what follows) $B_{\varepsilon}(x)$ denotes the closed ball with radius $\varepsilon$ around $x$. The following general stability result will serve as an orientation for the further development of our analysis.

Theorem 2.1 In addition to the general conditions, assume that

(i) $X$ is a CLM set for $\mathbf{P}(\mu)$ with respect to a bounded set $V$ (i.e., $X=\Psi_{V}(\mu)$ and $X$ is compact),

(ii) $g$ is locally Lipschitz continuous,

(iii) the probabilistic constraint function $\theta_{\mu}(\cdot)-p$ is metrically regular with respect to $C$ at each $x^{0} \in X$.

Then there are constants $L>0$ and $\delta>0$ such that the set-valued mapping $\Psi_{V}$ from $\left(\mathcal{P}\left(\mathbb{R}^{s}\right), \alpha\right)$ to $\mathbb{R}^{m}$ is upper semicontinuous at $\mu, \Psi_{V}(\nu)$ is a CLM set for $\mathbf{P}(\nu)$ with respect to $V$ and $\left|\varphi_{V}(\mu)-\varphi_{V}(\nu)\right| \leq L \cdot \alpha(\mu, \nu)$ holds whenever $\nu \in \mathcal{P}\left(\mathbb{R}^{s}\right), \alpha(\mu, \nu)<\delta$.

If, moreover, the following quadratic growth condition is satisfied

(iv) there exists a constant $c>0$ such that we have

$$
g(x) \geq \varphi_{V}(\mu)+c \cdot \operatorname{dist}\left(x, \Psi_{V}(\mu)\right)^{2} \forall x \in M_{p}(\mu) \cap V,
$$

then $\Psi_{V}$ is upper Hölder continuous at $\mu$ with rate $1 / 2$, i.e.,

$$
\sup _{x \in \Psi_{V}(\nu)} \operatorname{dist}\left(x, \Psi_{V}(\mu)\right) \leq L \cdot \alpha(\mu, \nu)^{1 / 2} \text { whenever } \nu \in \mathcal{P}\left(\mathbb{R}^{s}\right), \alpha(\mu, \nu)<\delta .
$$

\section{Proof:}

The first part of the assertion is proved in Theorem 3.2 of [44]. It remains to note that condition (iii) is equivalent to the fact that the set-valued mapping $q \mapsto M_{q}(\mu)$ from $R^{d}$ to $\mathbb{I R}^{m}$ is pseudo-Lipschitzian at each pair $\left(x^{0}, p\right) \in X \times\{p\}$ (cf. [35], Theorem 1.5). On the other hand, the latter property is equivalent to the local Lipschitz continuity of the function $(x, q) \mapsto \operatorname{dist}\left(x, M_{q}(\mu)\right)$ from $\mathbb{R}^{m} \times \mathbb{R}^{d}$ to $\mathbb{R}$ at each $\left(x^{0}, p\right) \in X \times\{p\}$ (see Theorem 2.3 in [40]), which is assumed in [44]. The second part of the result follows from Theorem 2.2 in 
[26] by using the same arguments as by deriving Theorem 3.2 in [44] from Proposition 1 and Theorem 1 in [25] (see also Theorem 2.5 in [42]).

All assumptions (i)-(iv) in the theorem concern the original (or unperturbed) problem $\mathbf{P}(\mu)$. While (i) and (ii) do not require further discussion, the conditions (iii) and (iv) are decisive and deserve verification. Verifiable conditions for the quadratic growth condition are apparently not yet known. In Section 4 we shall take up this question for the case of a single (joint) probabilistic constraint (i.e., $\mathrm{d}=1$ ), polyhedral $C$ and a set-valued mapping $H$ having a convex polyhedral graph. The method for proving the corresponding result (Theorem 4.2) will even allow to establish a Hausdorff Hölder continuity result for $\Psi_{V}$. The metric regularity property (iii) (or its equivalent characterizations mentioned in the above proof) has already been discussed in some special cases for $C$ and for $H_{j}(j=1, \ldots, d)$ (cf. [42], [43], [44], [46]). In Section 3 we shall considerably extend earlier results in this direction by exploiting recent tools from nonsmooth analysis. In order to explain this extension, we record now a metric regularity result for a situation where the data satisfy certain convexity properties. For this purpose and for later use we introduce the notion of an $r$-concave probability measure $(r \in[-\infty, \infty])$. Following [10] and [12] we define the generalized mean function $m_{r}$ on $\mathbb{R}_{+} \times \mathbb{R}_{+} \times[0,1]$ as follows:

$$
m_{r}(a, b ; \lambda)=\left\{\begin{array}{cl}
\left(\lambda a^{r}+(1-\lambda) b^{r}\right)^{1 / r} & \text { if } r \in(0, \infty) \text { or } r \in(-\infty, 0), a b>0 \\
0 & \text { if } a b=0, r \in(-\infty, 0) \\
a^{\lambda} b^{1-\lambda} & \text { if } r=0 \\
\max \{a, b\} & \text { if } r=\infty \\
\min \{a, b\} & \text { if } r=-\infty
\end{array}\right.
$$

The measure $\mu \in \mathcal{P}\left(\mathbb{R}^{s}\right)$ is called $r$-concave, $r \in[-\infty, \infty]([8],[12],[38])$, if the inequality $\mu\left(\lambda B_{1}+(1-\lambda) B_{2}\right) \geq m_{r}\left(\mu\left(B_{1}\right), \mu\left(B_{2}\right) ; \lambda\right)$ holds for all $\lambda \in[0,1]$ and all Borel subsets $B_{1}, B_{2}$ of $\mathbb{R}^{s}$ such that $\lambda B_{1}+(1-\lambda) B_{2}$ is Borel. For $r=0$ and $r=-\infty, \mu$ is also called logarithmic concave and quasi-concave, respectively $([37])$. Since $m_{r}(a, b ; \lambda)$ is increasing in $r$ if all the other variables are fixed, the sets of all $r$-concave probability measures are increasing if $r$ is decreasing. It is known (cf. [8], [10], [37], [38]) that $\mu \in \mathcal{P}\left(\mathbb{R}^{s}\right)$ is $r$-concave for some $r \in[-\infty, 1 / s]$ if $\mu$ has a density $f_{\mu}$ such that

$$
f_{\mu}(\lambda z+(1-\lambda) \tilde{z}) \geq m_{r(s)}\left(f_{\mu}(z), f_{\mu}(\tilde{z}) ; \lambda\right), \quad \text { where } r(s)=r(1-r s)^{-1}
$$

holds for all $\lambda \in[0,1]$ and $z, \tilde{z} \in \mathbb{R}^{s}$. A density $f_{\mu}$ satisfying (5) is called $r(s)$ - unimodal (see e.g. [12] for a detailed discussion of unimodality). We mention that e.g. the uniform distribution (on some bounded convex set), the (nondegenerate) multivariate normal distribution, the Dirichlet distribution, the multivariate Student and Pareto distributions are $r$-concave for some $r \in(-\infty, \infty]$ (see [8], Chapter 4 in [38]).

Proposition 2.2 In addition to the general assumptions, let $C$ be convex, $H_{j}(j=1, \ldots, d)$ have convex graphs and let $\mu$ be $r$-concave for some $r \in(-\infty, \infty]$. Suppose there exists an element $\bar{x} \in C$ such that the strict inequality $\theta_{\mu}(\bar{x})>p$ holds componentwise. Then the probabilistic constraint function $\theta_{\mu}(\cdot)-p$ is metrically regular with respect to $C$ at each feasible $x^{0} \in M_{p}(\mu)$. 
The proof is carried out in [44] (as part of the proof of Corollary 3.7) making use of the Robinson-Ursescu theorem for multifunctions having a closed convex graph. In Section 3 we shall study the case of $C \subseteq \mathbb{R}^{m}$ being closed and $H_{j}(x)=\left\{z \in \mathbb{R}^{s} \mid h_{j}(x) \geq z_{j}\right\}$ with $h_{j}: \mathbb{R}^{m} \rightarrow \mathbb{R}^{s_{j}}, j=1, \ldots, d$ and $\sum_{j=1}^{d} s_{j}=s$. For $d=1, C=\mathbb{R}^{m}$, continuously differentiable $h=h_{1}$ and a probability measure $\mu$ having locally Lipschitzian distribution function $F_{\mu}$, a particular metric regularity result is given in [42] (Corollary 5.6) using the Clarke generalized gradient. This has been partially extended by allowing for a general closed subset $C$ of $\mathbb{R}^{m}$ (but assuming $h$ to be linear) in [43] (Proposition 2.1) by making use of Clarke's nonsmooth calculus. Another type of result for a nonconvex situation (with $d=1, C$ convex, $h$ linear, but without assuming that $\mu$ has concavity properties) is developed in [46] (Theorem 4.6) and [43] (Corollary 2.2) by imposing a local growth condition on the composite function $F_{\mu}(h(\cdot)$ ) near binding feasible points. In Section 3 these earlier results will be unified and considerably extended by relaxing assumptions on the data and by relying on Mordukhovich's calculus for the approximate subdifferential and coderivative. A new local growth condition for the composite function $\mu\left(H_{j}(\cdot)\right)$ is identified and shown to be sufficient (and in some cases also necessary) for metric regularity (Theorem 3.12). Later, separate constraint qualifications for the measure and the function $H$ that imply metric regularity are established. Furthermore, the question of global metric regularity is discussed. This is of interest, since the set $X$ in condition (iii) of Theorem 2.1 is not explicitly given in general. The constraint qualifications needed in [43] and [42] for the special cases considered there, are recovered from our general results.

Finally, we give an idea how a quantitative stability result like Theorem 2.1 can be employed to derive asymptotic properties of solutions to $\mathbf{P}(\mu)$ when estimating $\mu$ by empirical measures. Let $\xi_{1}, \xi_{2}, \ldots, \xi_{n}, \ldots$ be independent $\mathbb{I R}^{s}$ - valued random variables on some probability space $(\Omega, \mathcal{A}, P)$ having common distribution $\mu$. The empirical measure of $\xi_{1}, \ldots, \xi_{n}$ is $\mu_{n}=n^{-1} \sum_{i=1}^{n} \delta_{\xi_{i}}(n \in \mathbb{N})$, where $\delta_{z}$ is the probability measure with mass one at $z \in \mathbb{R}^{s}$. The following result represents a large deviation type estimate for the 'distance'

$$
\sup _{x \in \Psi_{V}\left(\mu_{n}\right)} \operatorname{dist}\left(x, \Psi_{V}(\mu)\right)
$$

of sets of local solutions to $\mathbf{P}\left(\mu_{n}\right)$ and $\mathbf{P}(\mu)$, respectively, as $n$ tends to infinity. We note that this 'distance' is an extended real-valued $\mathcal{A}$-measurable mapping due to Theorem $2 \mathrm{~K}$ in [41]. To state the result we need the notion of a permissible Vapnik-Chervonenkis (VC) class of sets. A collection $\mathcal{C}$ of sets is said to shatter a set $A$ if every subset of $A$ is of the form $B \cap A$ for some $B \in \mathcal{C}$. The supremum of cardinalities of finite sets shattered by $\mathcal{C}$ is denoted by $s(\mathcal{C}) . \mathcal{C}$ is called a $\mathrm{VC}$ class iff $s(\mathcal{C})$ is finite. We refer to [13], [50] for a detailed discussion of VC classes and empirical measures. A class $\mathcal{C}$ of Borel subsets of $\mathbb{R}^{s}$ is called permissible iff $\sup _{B \in \mathcal{C}}\left|\mu(B)-\mu_{n}(B)\right|$ is a (real-valued) $\mathcal{A}$-measurable function.

Proposition 2.3 Assume that the conditions (i)-(iv) of Theorem 2.1 are satisfied and that $\mathcal{B}$ forms a permissible VC class of closed subsets of $\mathbb{I R}^{s}$. Then it holds for all $\varepsilon>0$ that

$$
\limsup _{n \rightarrow \infty} n^{-1} \log P\left(\sup _{x \in \Psi_{V}\left(\mu_{n}\right)} \operatorname{dist}\left(x, \Psi_{V}(\mu)\right) \geq \varepsilon\right) \leq-2 \min \left\{\delta^{2}, \varepsilon^{4} L^{-4}\right\},
$$

where $L$ and $\delta$ denote the constants and $V$ the bounded open set arising in Theorem D.1. 


\section{Proof:}

Let $\varepsilon>0$ and $L, \delta$ and $V$ be as in Theorem 2.1. We set $A_{\delta}=\left\{\omega \mid \alpha\left(\mu, \mu_{n}\right)<\delta\right\}$ and know that $A_{\delta}$ belongs to $\mathcal{A}$ since $\mathcal{B}$ is permissible. With $\bar{A}_{\delta}$ denoting the complement of $A_{\delta}$ we obtain the following inclusion from Theorem 2.1:

$$
\begin{aligned}
\left\{\omega \mid \sup _{x \in \Psi_{V}\left(\mu_{n}\right)} \operatorname{dist}\left(x, \Psi_{V}(\mu)\right) \geq \varepsilon\right\} & \subseteq \bar{A}_{\delta} \cup\left\{\omega \mid \varepsilon \leq L \alpha\left(\mu, \mu_{n}\right)^{1 / 2}\right\} \\
& \subseteq\left\{\omega \mid \alpha\left(\mu, \mu_{n}\right) \geq \min \left\{\delta, \varepsilon^{2} L^{-2}\right\} .\right.
\end{aligned}
$$

Furthermore, since $\mathcal{B}$ is a VC class of (Borel) subsets of $\mathbb{R}^{s}$, for all $\lambda>0$ and $n \geq 2$ there exists a constant $K(\lambda)$ such that $P\left(\alpha\left(\mu, \mu_{n}\right) \geq \lambda\right) \leq K(\lambda) p(n) \exp \left(-2 \lambda^{2} n\right)$, where the function $p$ has polynomial growth (cf. chapter 26 in [50]). Hence, we obtain

$$
n^{-1} \log P\left(\alpha\left(\mu, \mu_{n}\right) \geq \lambda\right) \leq n^{-1} \log (K(\lambda) p(n))-2 \lambda^{2}
$$

and

$$
\begin{aligned}
& \limsup _{n \rightarrow \infty} n^{-1} \log P\left(\sup _{x \in \Psi_{V}\left(\mu_{n}\right)} \operatorname{dist}\left(x, \Psi_{V}(\mu)\right) \geq \varepsilon\right) \leq \\
& \limsup _{n \rightarrow \infty} n^{-1} \log P\left(\alpha\left(\mu, \mu_{n}\right) \geq \min \left\{\delta, \varepsilon^{2} L^{-2}\right\}\right) \leq-2\left(\min \left\{\delta, \varepsilon^{2} L^{-2}\right\}\right)^{2} .
\end{aligned}
$$

Examples of VC classes are e.g. the collection of all $s$-dimensional intervals, all closed balls in $\mathbb{R}^{s}$, all half spaces in $\mathbb{R}^{s}$ and all polyhedra with at most $k$ faces $([13],[50])$. The notion 'permissible' for the required measurability property of $\alpha\left(\mu, \mu_{n}\right)$ is borrowed from [36]. In Appendix $\mathrm{C}$ of [36] general techniques are described to establish that classes of sets are permissible. Another idea is to require a separability assumption on the process $\left\{\mu_{n}(B)-\mu(B) \mid B \in \mathcal{B}\right\}$. It is known, for example, that $\mathcal{B}$ is permissible iff it is universally separable (cf. Chapter II in $[36]) . \mathcal{B}$ is called universally separable iff there exists a countable subclass $\mathcal{B}_{0}$ such that each characteristic function $\chi_{B}$ with $B \in \mathcal{B}$ is the pointwise limit of a sequence $\left(\chi_{B_{n}}\right)$ with $B_{n} \in \mathcal{B}_{0}$. In particular, the class of closed convex subsets of $\mathbb{R}^{s}$ is universally separable.

\section{Metric regularity of probabilistic constraints}

The importance of metric regularity as a stability concept in stochastic programming has been outlined in Section 2 (Theorem 2.1). In this section we study a specific class of probabilistic constraints by putting

$$
H_{j}(x)=\left\{z \in \mathbb{R}^{s} \mid h_{j}(x) \geq z_{j}\right\} \quad x \in \mathbb{R}^{m} ; j=1, \ldots, d
$$

in the general model $\mathbf{P}(\mu)$ formulated in Section 1. Here we assume that $z_{j} \in \mathbb{R}^{s_{j}}, h_{j}: \mathbb{R}^{m} \rightarrow$ $\mathbb{R}^{s_{j}}, z=\left(z_{1}, \ldots, z_{d}\right) \in \mathbb{R}^{s}=\mathbb{R}^{s_{1}} \times \cdots \times \mathbb{R}^{s_{d}}$. Then the probabilistic constraint becomes

$$
M=\left\{x \in C \mid \mu\left(\left\{z \in \mathbb{R}^{s} \mid h_{j}(x) \geq z_{j}\right\}\right) \geq p_{j}\right\} \quad(j=1, \ldots, d),
$$


where $C \subseteq \mathbb{R}^{m}$ is closed, $\mu \in \mathcal{P}\left(\mathbb{R}^{s}\right)$ is a probability measure on $R^{s}$ and $p_{j} \in(0,1)$ are prescribed probability levels. For the following it will be more convenient to transform (6) into the equivalent description

$$
M=\left\{x \in C \mid \Phi_{\mu}(h(x)) \geq p\right\},
$$

where $h=\left(h_{1}, \ldots, h_{d}\right): \mathbb{R}^{m} \rightarrow \mathbb{R}^{s}$ and $p=\left(p_{1}, \ldots, p_{d}\right)$ refer to the entities introduced above. The mapping $\Phi_{\mu}=\left(\Phi_{\mu}^{1}, \ldots, \Phi_{\mu}^{d}\right): \mathbb{R}^{s} \rightarrow \mathbb{R}^{d}$ comprises the marginal distribution functions of $\mu$ as its components:

$$
\Phi_{\mu}^{j}(y)=F_{\mu}\left(\infty, \ldots, \infty, \frac{j}{y_{j}}, \infty, \ldots, \infty\right) \quad(j=1, \ldots, d),
$$

where $y=\left(y_{1}, \ldots, y_{d}\right) \in \mathbb{R}^{s}, y_{j} \in \mathbb{R}^{s_{j}}(j=1, \ldots, d)$. Note that $\Phi_{\mu}$ is a non-decreasing mapping which, in case of $d=1$, reduces to the usual distribution function $F_{\mu}$. In particular, the components $\Phi_{\mu}^{j}$ are upper semicontinuous.

According to the remarks following Proposition 2.2 the aim of this section is to formulate sufficient characterizations of metric regularity in a general nonsmooth framework. As the main tool the subdifferential calculus by Mordukhovich [33] shall be applied. This offers certain advantages over using the corresponding (larger in general) concepts by Clarke [11]. In particular, the Mordukhovich coderivative yields an equivalent criterion for metric regularity [32]. It turns out that, for instance in the case of a single locally Lipschitzian inequality $f(x) \leq 0$, which is binding at some feasible point $\bar{x}$, an equivalent characterization of metric regularity by a relation like $0 \notin \partial f(\bar{x})$ requires the departure of $\partial$ from the framework of convexity. In fact, it is shown in [17] that Mordukhovich's subdifferential of Lipschitzian functions may be homeomorphic to any compact subset of $\mathbb{R}^{n}$, in particular, it may contain an arbitrary number of connected components (for a related extension to the more general Hilbert space setting see $[18])$.

\subsection{Basics from nonsmooth analysis}

In this section, some basic concepts for characterizing metric regularity in a nonsmooth setting shall be recalled. Let $X, Y, Z$ be arbitrary sets. For multifunctions $\Phi: X \underset{\rightarrow}{\rightarrow} Y, \Theta: Y \rightarrow{ }_{\rightarrow} Z$ put

$$
\begin{aligned}
\operatorname{Ker} \Phi & =\{x \in X \mid 0 \in \Phi(x)\} \\
\operatorname{Im} \Phi & =\{y \in Y \mid y \in \Phi(x), x \in X\} \\
\operatorname{Gph} \Phi & =\{(x, y) \in X \times Y \mid y \in \Phi(x)\} \\
\Phi^{-1}(y) & =\{x \in X \mid y \in \Phi(x)\} \\
\Theta \circ \Phi(x) & =\bigcup \Theta(y) \quad(x \in X), \text { and if } X=\mathbb{R}^{n_{1}}, Y=\mathbb{R}^{n_{2}}: \\
\limsup _{x \rightarrow x^{0}} \Phi(x) & =\left\{y \in Y \mid \exists x_{n} \rightarrow x^{0} \exists y_{n} \rightarrow y: y_{n} \in \Phi\left(x_{n}\right)\right\} .
\end{aligned}
$$

Now let $X, Y$ be two normed spaces. A multifunction $\Phi: X \rightarrow Y$ is called metrically regular at some point $\left(x^{0}, y^{0}\right) \in \mathrm{Gph} \Phi$ if there are constants $a>0$ and $\varepsilon>0$ such that

$$
\operatorname{dist}\left(x, \Phi^{-1}(y)\right) \leq a \cdot \operatorname{dist}(y, \Phi(x)) \quad \forall(x, y) \in B_{\varepsilon}\left(x^{0}\right) \times B_{\varepsilon}\left(y^{0}\right) .
$$


The abstract form of constraint sets writes as $C \cap F^{-1}(K)$, where $C \subseteq X$ and $K \subseteq Y$ are closed subsets of the respective spaces ( $K$ usually being a closed convex cone) and $F: X \rightarrow Y$ is the constraint function. Then, $F$ is said to be metrically regular with respect to $C$ at some feasible point $x^{0} \in C \cap F^{-1}(K)$ if the associated multifunction

$$
\Phi(x)=\left\{\begin{array}{cl}
-F(x)+K & \text { for } x \in C \\
\emptyset & \text { else }
\end{array}\right.
$$

is metrically regular at $\left(x^{0}, 0\right)$. It is easily seen that this is equivalent to the conventional definition of metric regularity for constrained systems:

$\exists \varepsilon>0 \exists a>0 \forall(x, y) \in\left(C \cap B_{\varepsilon}\left(x^{0}\right)\right) \times B_{\varepsilon}(0): \quad \operatorname{dist}\left(x, C \cap F^{-1}(K-y)\right) \leq a \cdot \operatorname{dist}(F(x), K-y)$

Note that in this relation only the constraints given by $F$ are subject to perturbations $y$ whereas $C$ is considered to be a fixed set of unperturbed constraints.

For some closed subset $S \subseteq \mathbb{R}^{n}$ and $x^{0} \in S$ the following concepts are defined:

$$
\begin{aligned}
T\left(S ; x^{0}\right)= & \limsup _{\substack{t \downarrow 0 \\
T_{c}}} t^{-1}\left(S-\left\{x^{0}\right\}\right) \quad \text { (contingent cone) } \\
T_{c}\left(S ; x^{0}\right)= & \left\{h \in \mathbb{R}^{n} \mid \forall x_{n} \rightarrow x^{0}\left(\left\{x_{n}\right\} \subseteq S\right) \forall t_{n} \downarrow 0 \exists h_{n} \rightarrow h: x_{n}+t_{n} h_{n} \in S\right\} \\
& \text { (Clarke's tangent cone) } \\
T^{0}\left(S ; x^{0}\right)= & \left\{x^{*} \in \mathbb{R}^{n} \mid\left\langle x^{*}, h\right\rangle \leq 0 \forall h \in T\left(S ; x^{0}\right)\right\} \quad \text { (Fréchet normal cone) } \\
N_{a}\left(S ; x^{0}\right)= & \limsup _{\substack{x \rightarrow x^{0} \\
x \in S}} T^{0}(S ; x) \quad \text { (approximate normal cone) } \\
N_{c}\left(S ; x^{0}\right)= & \left\{x^{*} \in \mathbb{R}^{n} \mid\left\langle x^{*}, h\right\rangle \leq 0 \forall h \in T_{c}\left(S ; x^{0}\right)\right\} \quad \text { (Clarke's normal cone) }
\end{aligned}
$$

The normal cone $N_{a}$ induces the approximate subdifferential for lower semicontinuous functions $f: \mathbb{R}^{n} \rightarrow \mathbb{R}:$

$$
\partial_{a} f\left(x^{0}\right)=\left\{x^{*} \in \mathbb{R}^{n} \mid\left(x^{*},-1\right) \in N_{a}\left(\text { Epi } f ;\left(x^{0}, f\left(x^{0}\right)\right)\right)\right\},
$$

where Epi refers to the epigraph. For locally Lipschitzian functions Clarke's subdifferential $\partial_{c}$ relates to $\partial_{a}$ as

$$
\partial_{c} f\left(x^{0}\right)=\overline{\operatorname{conv}} \partial_{a} f\left(x^{0}\right) .
$$

A closed subset $S \subseteq \mathbb{R}^{n}$ is called regular at $x^{0} \in S$ in the sense of Clarke, if $T\left(S ; x^{0}\right)=$ $T_{c}\left(S ; x^{0}\right)$. Similarly, a locally Lipschitzian function $f$ is called regular at $x^{0} \in \mathbb{R}^{n}$ in the sense of Clarke, if $T$ (Epi $\left.f ;\left(x^{0}, f\left(x^{0}\right)\right)\right)=T_{c}$ (Epi $\left.f ;\left(x^{0}, f\left(x^{0}\right)\right)\right)$. In case of the mentioned kinds of regularity it holds that $N_{c}\left(S ; x^{0}\right)=N_{a}\left(S ; x^{0}\right)$ and $\partial_{c} f\left(x^{0}\right)=\partial_{a} f\left(x^{0}\right)$.

A multifunction $\Phi: \mathbb{R}^{n \rightarrow} \rightarrow \mathbb{R}^{m}$ with closed graph and some point $\left(x^{0}, y^{0}\right) \in \mathrm{Gph} \Phi$ induces a multifunction $D_{a}^{*} \Phi\left(x^{0}, y^{0}\right): \mathbb{R}_{\rightarrow}^{m} \rightarrow R^{n}$ defined via

$$
D_{a}^{*} \Phi\left(x^{0}, y^{0}\right)\left(y^{*}\right)=\left\{x^{*} \in \mathbb{R}^{n} \mid\left(x^{*},-y^{*}\right) \in N_{a}\left(\operatorname{Gph} \Phi ;\left(x^{0}, y^{0}\right)\right)\right\},
$$

which is called the approximate coderivative of $\Phi$ at $\left(x^{0}, y^{0}\right)$. For single valued, locally Lipschitzian functions $\Phi: \mathbb{R}^{n} \rightarrow \mathbb{R}^{m}$ one has (see [19], Prop. 8):

$$
D_{a}^{*} \Phi(x, \Phi(x))\left(y^{*}\right)=\partial_{a}\left\langle y^{*}, \Phi\right\rangle(x) \quad \forall x \in \mathbb{R}^{n} \forall y^{*} \in \mathbb{R}^{m}
$$


The following results are due to Mordukhovich (compare [32], [33]) and will be substantially exploited in this section:

Theorem 3.1 A multifunction $\Phi: \mathbb{R}^{n \rightarrow} \mathbb{I R}^{m}$ with closed graph is metrically regular at some point $\left(x^{0}, y^{0}\right) \in \operatorname{Gph} \Phi$ if and only if $\operatorname{Ker} D_{a}^{*} \Phi\left(x^{0}, y^{0}\right)=\{0\}$.

Theorem 3.2 Let the multifunctions $\Phi: \mathbb{R}^{n} \rightarrow \mathbb{R}^{m}$ and $\Theta: \mathbb{R}^{m} \rightarrow \mathbb{R}^{k}$ have closed graph and $(\bar{x}, \bar{z}) \in \operatorname{Gph}(\Theta \circ \Phi)$. Suppose that the multifunction $M: \mathbb{R}^{n} \times \mathbb{R}^{\vec{k}} \rightarrow \mathbb{R}^{m}$ defined by

$$
M(x, z)=\Phi(x) \cap \Theta^{-1}(z)
$$

is locally bounded around $(\bar{x}, \bar{z})$ and that the condition

$$
D_{a}^{*} \Theta(y, \bar{z})(0) \cap \operatorname{Ker} D_{a}^{*} \Phi(\bar{x}, y)=\{0\} \quad \forall y \in M(\bar{x}, \bar{z})
$$

holds. Then one has

$$
D_{a}^{*}(\Theta \circ \Phi)(\bar{x}, \bar{z})\left(z^{*}\right) \subseteq \bigcup_{y \in \Phi(\bar{x}) \cap \Theta^{-1}(\bar{z})} D_{a}^{*} \Phi(\bar{x}, y) \circ D_{a}^{*} \Theta(y, \bar{z})\left(z^{*}\right) \quad \forall z^{*} \in \mathbb{R}^{k}
$$

Lemma 3.3 Let $S_{1}, S_{2} \subseteq \mathbb{R}^{n}$ be closed sets with $\bar{x} \in S_{1} \cap S_{2}$ and $N_{a}\left(S_{1} ; \bar{x}\right) \cap-N_{a}\left(S_{2} ; \bar{x}\right)=$ $\{0\}$. Then

$$
N_{a}\left(S_{1} \cap S_{2} ; \bar{x}\right) \subseteq N_{a}\left(S_{1} ; \bar{x}\right)+N_{a}\left(S_{2} ; \bar{x}\right),
$$

where equality holds if $S_{1}, S_{2}$ are regular in the sense of Clarke.

\subsection{An explicit growth condition}

Before dealing with the chance constraint (7) we start our considerations with general constraint sets described by finitely many inequalities:

$$
P=\{x \in C \mid F(x) \geq 0\} \quad F: \mathbb{R}^{n} \rightarrow \mathbb{R}^{k}, C \subseteq \mathbb{R}^{n}(C \text { closed }) .
$$

Obviously, (7) fits into this type of constraints. For a feasible point $x^{0} \in P$ denote by

$$
\begin{aligned}
& I=\left\{i \in\{1, \ldots, k\} \mid F_{i}\left(x^{0}\right)=0\right\} \\
& J=\left\{i \in\{1, \ldots, k\} \mid F_{i} \text { is not continuous at } x^{0}\right\}
\end{aligned}
$$

the sets of active and noncontinuity indices, respectively, at $x^{0}$, where the $F_{i}$ refer to the components of $F$. The following definition provides an explicit growth condition on the components of $F$ which will imply metric regularity.

Definition 3.4 We say that the constraint mapping $F: \mathbb{R}^{n} \rightarrow \mathbb{R}^{k}$ in (10) is growing at some feasible point $x^{0} \in P$ with respect to $C$ if

(i) $F_{i}$ is upper semicontinuous in a neighbourhood of $x^{0}$ for $i \in\{1, \ldots, k\}$ 
(ii) there exists an $\rho>0$ such that the following local growth condition is fulfilled:

$$
\exists \eta>0 \forall x \in B_{\eta}\left(x^{0}\right) \cap C \forall \varepsilon>0 \exists y \in B_{\varepsilon}(x) \cap C: F_{i}(y)>F_{i}(x)+\rho\|y-x\| \quad \forall i \in I \cup J .
$$

Note that, for continuous $F$, this is merely a growth condition imposed on the active components at $x^{0}$.

Lemma 3.5 Let $x^{0} \in P$ be a feasible point of (10). If $F$ is growing at $x^{0}$ with respect to $C$, then $F$ is metrically regular at $x^{0}$ with respect to $C$.

\section{Proof:}

According to Section 3.1 one has to verify metric regularity of the multifunction

$$
\Phi(x)=\left\{\begin{array}{cl}
-F(x)+\mathbb{R}_{+}^{k} & \text { if } x \in C \\
\emptyset & \text { else }
\end{array}\right.
$$

at the point $\left(x^{0}, 0\right) \in \operatorname{Gph} \Phi$. Choose a number $\gamma$ with $0<\gamma<\eta$ (where $\eta$ refers to Definition $3.4)$ which, according to the definition of the index sets $I$ and $J$, satisfies

$$
F_{i}(z)>\gamma \quad \forall i \notin I \cup J \forall z \in \operatorname{int} B_{\gamma}\left(x^{0}\right)
$$

For computing Fréchet normal cones $T^{0}$ in a neighbourhood of $\left(x^{0}, 0\right)$, fix an arbitrary $(x, b) \in$ (int $B_{\gamma}\left(x^{0}\right) \times$ int $\left.B_{\gamma}(0)\right) \cap \mathrm{Gph} \Phi$. Then $x \in C$ and $b \geq-F(x)$ by definition of $\Phi$.

Let us first consider the case $I \cup J \neq \emptyset$. By Definition 3.4 there exists a sequence $y_{l} \rightarrow x$ $\left(y_{l} \in C\right)$, such that $F_{i}\left(y_{l}\right)>F_{i}(x)+\rho\left\|y_{l}-x\right\| \forall i \in I \cup J$. Clearly $y_{l} \neq x$. We show that the vector

$$
\left(\begin{array}{l}
x \\
b
\end{array}\right)+\left\|y_{l}-x\right\|\left(\begin{array}{c}
\left(y_{l}-x\right) /\left\|y_{l}-x\right\| \\
-\rho \mathbf{1}
\end{array}\right)=\left(\begin{array}{c}
y_{l} \\
b-\rho\left\|y_{l}-x\right\| \mathbf{1}
\end{array}\right)
$$

with $\mathbf{1}=(1, \ldots, 1)^{T}$ belongs to Gph $\Phi$ for $l$ large enough. In fact, if $i \in I \cup J$, then

$$
[b]_{i}-\rho\left\|y_{l}-x\right\| \geq-F_{i}(x)-\rho\left\|y_{l}-x\right\|>-F_{i}\left(y_{l}\right)
$$

where the $[b]_{i}$ denote the corresponding components of $b$. On the other hand, taking for instance the Euclidean norm, $b \in \operatorname{int} B_{\gamma}(0)$ implies $[b]_{i}>-\gamma$, hence $[b]_{i}-\rho\left\|y_{l}-x\right\|>-\gamma$ for $i=1, \ldots, k$ and large $l$. In particular, relation (11) makes also the indices $i \notin I \cup J$ satisfy $[b]_{i}-\rho\left\|y_{l}-x\right\|>-F_{i}\left(y_{l}\right)$ ( $l$ large enough). Combining both cases one arrives at $b-\rho\left\|y_{l}-x\right\| \mathbf{1} \in-F\left(y_{l}\right)+\mathbb{R}_{+}^{k}$, which together with $y_{l} \in C$ yields $\left(y_{l}, b-\rho\left\|y_{l}-x\right\| \mathbf{1}\right) \in$ Gph $\Phi$. Without loss of generality, we assume $\left(y_{l}-x\right) /\left\|y_{l}-x\right\| \rightarrow \xi$, so (12) shows that $(\xi,-\rho \mathbf{1})$ belongs to the contingent cone $T(\mathrm{Gph} \Phi ;(x, b))$. Consequently,

$$
\left\langle(\xi,-\rho \mathbf{1}),\left(\xi^{*}, y^{*}\right)\right\rangle=\left\langle\xi, \xi^{*}\right\rangle-\rho\left\langle\mathbf{1}, y^{*}\right\rangle \leq 0 \quad \forall\left(\xi^{*}, y^{*}\right) \in T^{0}(\operatorname{Gph} \Phi ;(x, b))
$$

Due to $\|\xi\|=1$ this means $\left\|\xi^{*}\right\| \geq\left\langle-\xi, \xi^{*}\right\rangle \geq-\rho\left\langle\mathbf{1}, y^{*}\right\rangle$.

Now turn to the case $I \cup J=\emptyset$. Here $(x, b)+\delta(0,-\rho \mathbf{1}) \in \mathrm{Gph} \Phi$ for sufficiently small $\delta>0$ (compare (11) and recall $[b]_{i}>-\gamma$ for the components of $\left.b\right)$. So $(0,-\rho \mathbf{1}) \in T(\operatorname{Gph} \Phi ;(x, b))$, 
and applying an arbitrary normal vector $\left(\xi^{*}, y^{*}\right)$ to this provides the inequality $-\rho\left\langle\mathbf{1}, y^{*}\right\rangle \leq 0$. Summarizing, one has

$$
\begin{gathered}
-\rho\left\langle\mathbf{1}, y^{*}\right\rangle \leq\left\|\xi^{*}\right\| \\
\forall\left(\xi^{*}, y^{*}\right) \in T^{0}(\operatorname{Gph} \Phi ;(x, b)) \forall(x, b) \in\left(\operatorname{int} B_{\gamma}\left(x^{0}\right) \times \operatorname{int} B_{\gamma}(0)\right) \cap \operatorname{Gph} \Phi
\end{gathered}
$$

in any case. Consider any $z^{*} \in \operatorname{Ker} D_{a}^{*} \Phi\left(x^{0}, 0\right)$. Local upper semicontinuity of all components $F_{i}$ together with the closedness of $C$ imply the closedness (near $\left(x^{0}, 0\right)$ ) of Gph $\Phi$. By virtue of Theorem 3.1 the lemma is proved if we can show that $z^{*}=0$. By definition

$$
\left(0,-z^{*}\right) \in N_{a}\left(\operatorname{Gph} \Phi ;\left(x^{0}, 0\right)\right)=\limsup _{\substack{(x, b) \rightarrow\left(x^{0}, 0\right) \\(x, b) \in \operatorname{Gph} \Phi}} T^{0}(\operatorname{Gph} \Phi ;(x, b))
$$

so there are sequences

$$
\left(x_{l}, b_{l}\right) \rightarrow\left(x^{0}, 0\right),\left(x_{l}, b_{l}\right) \in \operatorname{Gph} \Phi,\left(\xi_{l}^{*}, y_{l}^{*}\right) \rightarrow\left(0,-z^{*}\right),\left(\xi_{l}^{*}, y_{l}^{*}\right) \in T^{0}\left(\operatorname{Gph} \Phi ;\left(x_{l}, b_{l}\right)\right) .
$$

Along with (13) this leads to $-\rho\left\langle\mathbf{1},-z^{*}\right\rangle \leq 0$, or, because $\rho$ is positive, to $\left\langle\mathbf{1}, z^{*}\right\rangle \leq 0$. On the other hand, $b_{l} \geq-F\left(x_{l}\right)$ implies $\left(0, e_{j}\right) \in T\left(\operatorname{Gph} \Phi ;\left(x_{l}, b_{l}\right)\right)$ for arbitrary standard unit vectors $e_{j} \in \mathbb{R}^{k},(j=1, \ldots, k)$, hence $y_{l}^{*} \leq 0$. By continuity, $z^{*} \geq 0$, so the desired relation $z^{*}=0$ follows.

The reverse direction of Lemma 3.5 does not hold in general, as one can see from the example $C=\mathbb{R}, F(x)=|x|$ if $x \neq 0$ and $F(0)=1$. While $F$ is upper semicontinuous, it fails to be growing at 0 . On the other hand one computes

$$
N_{a}(\operatorname{Epi}(-F) ;(0,0))=\left\{(x, y) \in \mathbb{R}^{2} \mid y \in\{0,-|x|\}\right\}
$$

hence, $\operatorname{Ker} D_{a}^{*} \phi(0,0)=\{0\}$ for the multifunction $\phi=-F+\mathbb{R}_{+}$, so $\phi$ is metrically regular at $(0,0)$ due to Theorem 3.1 and, therefore, $F$ is metrically regular at 0 .

For some special cases, however, the growth condition of Definition 3.4 is an equivalent characterization of metric regularity in the constraint system (10). Before establishing a corresponding result, we need some preparatory propositions, some of which are of independent interest.

Proposition 3.6 In the constraint set (10) assume that $k=1,-F$ is a regular, locally Lipschitzian function at some $x^{0} \in P$ with $F\left(x^{0}\right)=0$ and $C$ is a regular set at $x^{0}$ (here twice 'regular' is meant in the sense of Clarke). Then, metric regularity of $F$ at $x^{0}$ w.r.t. $C$ implies $\partial_{a}(-F)\left(x^{0}\right) \cap-N_{a}\left(C ; x^{0}\right)=\emptyset$

\section{Proof:}

Obviously, Epi $(-F)$ and $C \times \mathbb{R}$ are closed, regular subsets of $\mathbb{R}^{n+1}$, both of which contain $\left(x^{0}, 0\right)$. Since $F$ is locally Lipschitzian, one has $N_{a}\left(\operatorname{Epi}(-F) ;\left(x^{0}, 0\right)\right) \cap\left(\mathbb{R}^{n} \times\{0\}\right)=\{(0,0)\}$ (this follows from elementary calculations and amounts to the fact that the so-called singular subdifferential of locally Lipschitzian functions reduces to zero). Therefore

$$
\begin{aligned}
N_{a}\left(\operatorname{Epi}(-F) ;\left(x^{0}, 0\right)\right) \cap-N_{a}\left(C \times \mathbb{R} ;\left(x^{0}, 0\right)\right) & =N_{a}\left(\operatorname{Epi}(-F) ;\left(x^{0}, 0\right)\right) \cap\left[-N_{a}\left(C ; x^{0}\right) \times\{0\}\right] \\
& =\{(0,0)\}
\end{aligned}
$$


and Lemma 3.3 yields

$$
N_{a}\left(\operatorname{Epi}(-F) \cap(C \times \mathbb{R}) ;\left(x^{0}, 0\right)\right)=N_{a}\left(\operatorname{Epi}(-F) ;\left(x^{0}, 0\right)\right)+\left[N_{a}\left(C ; x^{0}\right) \times\{0\}\right] .
$$

Suppose there exists some $\xi \in \partial_{a}(-F)\left(x^{0}\right) \cap-N_{a}\left(C ; x^{0}\right)$. Then, according to the above decomposition

$$
(0,-1)=(\xi,-1)+(-\xi, 0) \in N_{a}\left(\operatorname{Epi}(-F) \cap(C \times \mathbb{R}) ;\left(x^{0}, 0\right)\right)=N_{a}\left(\operatorname{Gph} \phi ;\left(x^{0}, 0\right)\right),
$$

where $\phi: \mathbb{R}_{\rightarrow}^{n \rightarrow} \mathbb{R}$ is defined by $\phi(x)=-F(x)+\mathbb{R}_{+}$if $x \in C$, and $\phi(x)=\emptyset$ if $x \notin$ $C$. Hence, $1 \in \operatorname{Ker} D_{a}^{*} \phi\left(x^{0}, 0\right)$, so by Theorem $3.1 \phi$ is not metrically regular at $\left(x^{0}, 0\right)$. Consequently, $F$ is not metrically regular at $x^{0}$ w.r.t. $C$ in contradiction to the assumption. Thus $\partial_{a}(-F)\left(x^{0}\right) \cap-N_{a}\left(C ; x^{0}\right)=\emptyset$.

Proposition 3.7 In the constraint set (10) assume that $k=1$ and $F$ is a locally Lipschitzian function at some $x^{0} \in P$ with $F\left(x^{0}\right)=0$. Then the constraint qualification $\partial_{c} F\left(x^{0}\right) \cap$ $N_{c}\left(C ; x^{0}\right)=\emptyset$ implies $F$ to be growing at $x^{0}$ w.r.t. $C$.

\section{Proof:}

Since both $\partial_{c} F\left(x^{0}\right)$ and $N_{c}\left(C ; x^{0}\right)$ are closed, convex and nonempty and the subdifferential is even compact, the separation theorem provides the existence of some $h \in \mathbb{R}^{n},\|h\|=1$ and $\gamma \in \mathbb{R}$ such that

$$
\left\langle\xi^{*}, h\right\rangle<\gamma<\left\langle\pi^{*}, h\right\rangle \quad \forall\left(\xi^{*}, \pi^{*}\right) \in \partial_{c} F\left(x^{0}\right) \times N_{c}\left(C ; x^{0}\right) .
$$

¿From $\gamma<0$ (since $0 \in N_{c}\left(C ; x^{0}\right)$ ) and the cone property of $N_{c}$ one deduces $\left\langle\pi^{*}, h\right\rangle \geq 0$ for all $\pi^{*} \in N_{c}\left(C ; x^{0}\right)$. Therefore $-h$ belongs to the dual of $N_{c}\left(C ; x^{0}\right)$, which is Clarke's tangent cone $T_{c}\left(C, x^{0}\right)$. On the other hand, Clarke's directional derivative of $F$ at $x^{0}$ in direction $h$ computes as

$$
d^{c} F\left(x^{0} ; h\right)=\max \left\{\left\langle\xi^{*}, h\right\rangle \mid \xi^{*} \in \partial_{c} F\left(x^{0}\right)\right\}<\gamma<0 .
$$

Suppose that $F$ is not growing at $x^{0}$ w.r.t. $C$. Then there exist sequences $x^{l} \rightarrow x^{0}\left(x^{l} \in C\right)$ and $\varepsilon_{l}>0$ such that $F(y) \leq F\left(x^{l}\right)+l^{-1}\left\|y-x^{l}\right\| \quad \forall y \in B_{\varepsilon_{l}}\left(x^{l}\right) \cap C$. Putting $t_{l}=\frac{1}{2} \min \left\{\varepsilon_{l}, l^{-1}\right\}$ one has $t_{l} \downarrow 0$ and $F(y) \leq F\left(x^{l}\right)+l^{-1}\left\|y-x^{l}\right\| \quad \forall y \in B_{2 t_{l}}\left(x^{l}\right) \cap C$. The above mentioned fact that $-h \in T_{c}\left(C ; x^{0}\right)$ implies that, along with the sequences $\left\{x^{l}\right\},\left\{t_{l}\right\}$, there is a sequence $h^{l} \rightarrow h$ such that $x^{l}-t_{l} h^{l} \in C$ for $l \in \mathbb{N}$. So, for large $l$, we get $x^{l}-t_{l} h^{l} \in B_{2 t_{l}}\left(x^{l}\right) \cap C$ (recall that $\|h\|=1$ ). With $y_{l}=x_{l}-t_{l} h_{l}$ it follows $y_{l} \rightarrow x^{0}$ and $F\left(y_{l}\right) \leq F\left(x^{l}\right)+l^{-1} t_{l}\left\|h_{l}\right\|$. According to the definition of Clarke's directional derivative one arrives at

$$
\begin{aligned}
d^{c} F\left(x^{0} ; h\right) & =\limsup _{\substack{x \rightarrow x^{0} \\
t \downarrow 0}} t^{-1}(F(x+t h)-F(x)) \geq \limsup _{l \rightarrow \infty} t_{l}^{-1}\left(F\left(y^{l}+t_{l} h\right)-F\left(y_{l}\right)\right) \\
& =\limsup _{l \rightarrow \infty} t_{l}^{-1}\left(F\left(y^{l}+t_{l} h_{l}\right)-F\left(y_{l}\right)\right) \geq \limsup _{l \rightarrow \infty}-l^{-1}\left\|h^{l}\right\|=0
\end{aligned}
$$

(here, replacing $h$ by $h_{l}$ in the third estimation relies on $F$ being locally Lipschitzian). This is a contradiction to (14). 
In the following, a function $F: \mathbb{R}^{n} \rightarrow \mathbb{R}^{k}$ is called nondecreasing at $x^{0} \in \mathbb{R}^{n}$ if the relation $y \geq x \Rightarrow F(y) \geq F(x)$ (with the partial orders in $\mathbb{R}^{n}$ and $\mathbb{R}^{k}$, respectively) holds for all $x, y$ in some neighbourhood of $x^{0}$. In particular, the function $\Phi_{\mu}$ in (7) is nondecreasing at all $x \in \mathbb{R}^{s}$.

Proposition 3.8 Let $F: \mathbb{R}^{n} \rightarrow \mathbb{R}^{k}$ have upper semicontinuous components and be nondecreasing at $x^{0} \in \mathbb{R}^{n}$. Then the associated multifunction $\phi: \mathbb{R}_{\rightarrow}^{n \rightarrow} \mathbb{R}^{k}$ defined by $\phi(x)=$ $-F(x)+\mathbb{R}_{+}^{k}$ satisfies $\operatorname{Im} D_{a}^{*} \phi\left(x^{0}, y\right) \subseteq \mathbb{R}_{-}^{n} \forall y \in \phi\left(x^{0}\right)$.

\section{Proof:}

First note that Gph $\phi$ is closed due to the upper semicontinuity of $F$. Consider arbitrary $y \in \phi\left(x^{0}\right)$ and $\left(x^{*}, y^{*}\right) \in \mathbb{R}^{n} \times \mathbb{R}^{k}$ such that $x^{*} \in D_{a}^{*} \phi\left(x^{0}, y\right)\left(y^{*}\right)$. This means $\left(x^{*},-y^{*}\right) \in$ $N_{a}\left(\operatorname{Gph} \phi ;\left(x^{0}, y\right)\right)$ and, by definition, there are sequences $\left(x_{l}, y_{l}\right) \rightarrow\left(x^{0}, y\right),\left(\left(x_{l}, y_{l}\right) \in \operatorname{Gph} \phi\right)$ and $\left(x_{l}^{*},-y_{l}^{*}\right) \rightarrow\left(x^{*},-y^{*}\right)\left(\left(x_{l}^{*},-y_{l}^{*}\right) \in T^{0}\left(\operatorname{Gph} \phi ;\left(x_{l}, y_{l}\right)\right)\right)$. Since $F$ is nondecreasing at $x^{0}$, one has $\left(e_{j}, 0\right) \in T\left(\operatorname{Gph} \phi ;\left(x_{l}, y_{l}\right)\right)$ for all standard unit vectors $e_{j} \in \mathbb{R}^{n}$ and for all $l \in \mathbb{N}$. It follows that $\left\langle\left(x_{l}^{*},-y_{l}^{*}\right),\left(e_{j}, 0\right)\right\rangle=\left(x_{l}^{*}\right)_{j} \leq 0$ for $j=1, \ldots, n$, hence $x_{l}^{*} \leq 0$ and $x^{*} \in \mathbb{R}_{-}^{n}$, as desired.

Corollary 3.9 If $F: \mathbb{R}^{n} \rightarrow \mathbb{R}$ is upper semicontinuous and nondecreasing at $x^{0}$, then $\partial_{a}(-F)\left(x^{0}\right) \subseteq \mathbb{R}_{-}^{n}$. If, additionally, $F$ is locally Lipschitzian at $x^{0}$, then $\partial_{c}(-F)\left(x^{0}\right) \subseteq \mathbb{R}_{-}^{n}$ and

$$
B_{\varepsilon}(0) \cap \partial_{a}(-F)\left(x^{0}\right)=\emptyset \Longrightarrow B_{\varepsilon / \sqrt{n}}(0) \cap \partial_{c}(-F)\left(x^{0}\right)=\emptyset \quad \forall \varepsilon>0,
$$

where the balls are taken w.r.t. the Euclidean norm.

\section{Proof:}

First note that $-F$ is lower semicontinuous, so (compare Section 3.1)

$$
\partial_{a}(-F)\left(x^{0}\right)=D_{a}^{*} \phi\left(x^{0},-F\left(x^{0}\right)\right)(1) \subseteq \mathbb{R}_{-}^{n}
$$

with reference to Proposition 3.8. The next assertion follows from (8) since $R_{-}^{n}$ is closed and convex. Concerning (15) one has

$$
\partial_{a}(-F)\left(x^{0}\right) \subseteq \mathbb{R}_{-}^{n} \cap\left\{x^{*} \mid\left\|x^{*}\right\|_{1} \geq \operatorname{dist}\left(0, \partial_{a}(-F)\left(x^{0}\right)\right)\right\},
$$

where $\|\cdot\|_{1}$ refers to the sum norm and dist to the Euclidean distance. The right-hand side of this inclusion is closed and convex, hence, again by $(8)$, it also contains $\partial_{c}(-F)\left(x^{0}\right)$ and one obtains

$$
\left\|x^{*}\right\| \geq n^{-1 / 2}\left\|x^{*}\right\|_{1} \geq n^{-1 / 2} \operatorname{dist}\left(0, \partial_{a}(-F)\left(x^{0}\right)\right)>\varepsilon / \sqrt{n} \quad \forall x^{*} \in \partial_{c}(-F)\left(x^{0}\right) .
$$

Corollary 3.10 If $F: \mathbb{R}^{n} \rightarrow \mathbb{R}$ is a locally Lipschitzian distribution function of some random variable, then $\partial_{a} F(x), \partial_{c} F(x) \subseteq \mathbb{R}_{+}^{n} \forall x \in \mathbb{R}^{n}$ and $0 \in \partial_{a} F(x)$ if and only if $0 \in \partial_{c} F(x)$. 


\section{Proof:}

Repeat the proof of Proposition 3.8 with $\phi(x)=F(x)+\mathbb{R}_{+}^{k}$ to get $\operatorname{Im} D_{a}^{*} \phi\left(x^{0}, y\right) \subseteq \mathbb{R} \mathbb{R}_{+}^{n} \forall y \in$ $\phi\left(x^{0}\right)$. Using this, repeat the proof of Corollary 3.9 and replace $-F$ by $F$ and $\mathbb{R} \mathbb{R}_{-}^{n}$ by $\mathbb{R}_{+}^{n}$ constantly.

Now we are able to formulate conditions under which metric regularity implies growth according to Definition 3.4, such that both concepts are equivalent in view of Lemma 3.5.

Lemma 3.11 In (10), let $k=1$ and $F$ be locally Lipschitzian at some feasible point $x^{0} \in P$. Then metric regularity of $F$ at $x^{0}$ w.r.t. $C$ implies that $F$ is growing at $x^{0}$ w.r.t. C provided that one of the following conditions holds:

(i) $-F$ and $C$ are regular at $x^{0}$ in the sense of Clarke.

(ii) $F$ is nondecreasing at $x^{0}$ and $C=\mathbb{R}^{n}$.

\section{Proof:}

According to Definition 3.4 only the binding case $F\left(x^{0}\right)=0$ is of interest. Consider the first condition in the lemma. Proposition 3.6 yields $\partial_{a}(-F)\left(x^{0}\right) \cap-N_{a}\left(C ; x^{0}\right)=\emptyset$. The regularity assumptions make the corresponding concepts of approximate and Clarke subdifferentiation coincide, hence $\partial_{c}(-F)\left(x^{0}\right) \cap-N_{c}\left(C ; x^{0}\right)=\emptyset$. This, however, means that $\partial_{c} F\left(x^{0}\right) \cap N_{c}\left(C ; x^{0}\right)=$ $\emptyset$. Now apply Proposition 3.7. Concerning the second condition in the lemma note that metric regularity of $F$ at $x^{0}$ implies metric regularity of the multifunction $-F+R_{+}$at $\left(x^{0}, 0\right)=\left(x^{0}, F\left(x^{0}\right)\right)$ (recall $\left.C=\mathbb{R}^{n}\right)$. Then Theorem 3.1 provides

$$
\begin{aligned}
0 \notin D_{a}^{*}\left(-F+\mathbb{R}_{+}\right)\left(x^{0}, 0\right)(1) & =\left\{x^{*} \in \mathbb{R}^{n} \mid\left(x^{*},-1\right) \in N_{a}\left(\operatorname{Epi}(-F) ;\left(x^{0}, F\left(x^{0}\right)\right)\right)\right\} \\
& =\partial_{a}(-F)\left(x^{0}\right) .
\end{aligned}
$$

¿From (15) we conclude that $0 \notin \partial_{c}(-F)\left(x^{0}\right)$, so $0 \notin \partial_{c} F\left(x^{0}\right)$. Apply Proposotion 3.7 (with $C=\mathbb{R}^{n}$, so $\left.N_{c}\left(C ; x^{0}\right)=\{0\}\right)$ once more.

Now we apply the above results to the characterization of metric regularity of the probabilistic constraint (7).

Theorem 3.12 In the probabilistic constraint ( 7$)$ let $h$ be continuous and $x^{0} \in M$ some feasible point. Suppose there exist $\rho>0, \eta>0$ such that for all components $\Phi_{\mu}^{j}$ of $\Phi_{\mu}$ that are not continuous at $h\left(x^{0}\right)$ or that are binding (i.e., $\left.\Phi_{\mu}^{j}\left(h\left(x^{0}\right)\right)=p_{j}\right)$ the growth condition

$$
\forall x \in B_{\eta}\left(x^{0}\right) \cap C \forall \varepsilon>0 \exists y \in B_{\varepsilon}(x) \cap C: \quad \Phi_{\mu}^{j}(h(y))>\Phi_{\mu}^{j}(h(x))+\rho\|y-x\|
$$

is fulfilled. Then the constraint function $\Phi_{\mu}(h(\cdot))-p$ is metrically regular at $x^{0}$ w.r.t. C. Next consider the special case $d=1$ (i.e., $\Phi_{\mu}=F_{\mu}$ ), where $h$ at $x^{0}$ and $F_{\mu}$ at $h\left(x^{0}\right)$ are locally Lipschitzian and where, additionally, one of the following conditions holds:

(i) $-F_{\mu} \circ h$ as a function and $C$ as a set are regular at $x^{0}$ in the sense of Clarke.

(ii) $h$ is nondecreasing at $x^{0}$ and $C=\mathbb{R}^{n}$. 
Then one has equivalence between metric regularity of the constraint function $F_{\mu}(h(\cdot))-p$ at $x^{0}$ w.r.t. $C$ and the following growth condition: If $F_{\mu}\left(h\left(x^{0}\right)\right)=p$, then there exist $\rho>0, \eta>0$ such that

$$
\forall x \in B_{\eta}\left(x^{0}\right) \cap C \forall \varepsilon>0 \exists y \in B_{\varepsilon}(x) \cap C: \quad F_{\mu}(h(y))>F_{\mu}(h(x))+\rho\|y-x\|
$$

\section{Proof:}

Recall that the components of $\Phi_{\mu}$ are automatically upper semicontinuous, hence the composition $\Phi_{\mu}(h(\cdot))-p$ enjoys the same property. Apply Lemma 3.5. The necessity of metric regularity with the growth condition in the second assertion is a special case of the first assertion. For the sufficiency apply Lemma 3.11 to the function $F=F_{\mu}(h(\cdot))-p$ and note that $F$ is nondecreasing at $x^{0}$ in the case (ii) since $F_{\mu}$ is always nondecreasing everywhere.

To illustrate the potential and the limitations of Theorem 3.12 consider the following two examples.

Example 3.13 In the probabilistic constraint (7) let $m=2, s=d=1, p=0.5, h\left(x_{1}, x_{2}\right)=$ $x_{1}+x_{2}$. Let $\mu$ be the uniform distribution over the interval $[-0.5,0.5]$ and take

$$
C=\left\{\left(x_{1}, x_{2}\right) \in \mathbb{R}^{2} \mid x_{1} \geq 0, x_{1}^{3} \leq x_{2} \leq x_{1}^{2}\right\}
$$

Obviously one has

$$
\Phi_{\mu}(y)=F_{\mu}(y)=y+0.5 \quad \forall y \in(-0.5,0.5) .
$$

The point of interest is $x^{0}=(0,0) \in C$. Then, in a small neighbourhood of this point, it holds that $F_{\mu}\left(h\left(x_{1}, x_{2}\right)\right)=x_{1}+x_{2}+0.5$. In particular, the constraint is binding at $x^{0}$. Being an affine linear function, $-F_{\mu} \circ h$ is regular at $x^{0}$ in the sense of Clarke. Furthermore, $T_{c}\left(C ; x^{0}\right)=T\left(C ; x^{0}\right)$, hence $C$ is a regular set at $x^{0}$ in the sense of Clarke. Therefore, the assumptions of the second assertion in Theorem 3.12 are fulfilled, so we know that checking metric regularity is equivalent to verifying the second growth condition in Theorem 3.12. Now, fix any $x \in C$ near $x^{0}$. One may find a point $y \in C, y \neq x$ arbitrarily close to $x$ such that $y-x \in \mathbb{R}_{+}^{2}$. Then the difference $F_{\mu}(h(y))-F_{\mu}(h(x))$ equals the sum norm $\|y-x\|_{1}$, therefore $F_{\mu} \circ h$ is growing at $x^{0}$ w.r.t $C$, hence metric regularity of $F_{\mu} \circ h$ holds at $x^{0}$ w.r.t. $C$.

In [43] (Corollary 2.2) a sufficient growth condition for metric regularity of the constraint function $\Phi_{\mu}(h(\cdot))-p$ was proposed for the special case $d=1, \Phi_{\mu}=F_{\mu}$ continuous, $h$ linear and $C$ convex. Essentially, growth was required along line segments in $C$. Note that in Example 3.13 there are no (nontrivial) line segments emanating from $x^{0}$ and entirely contained in $C$, so the mentioned condition does not work here although, apart from nonconvexity of $C$, the remaining assumptions are fulfilled. Furthermore, even if $C$ is convex and $F_{\mu}$ continuous, but $h$ slightly violates linearity (e.g. being piecewise differentiable), this condition does no longer hold true. This illustrates the extension obtained by Theorem 3.12.

The following example indicates a situation where metric regularity of chance constraints cannot be recovered from the growth condition of Theorem 3.12 (compare Remark 2.5 in [43]). 
Example 3.14 In (7), let $d=1$ and $\mu \in \mathcal{P}\left(\mathbb{R}^{s}\right)$ be a discrete measure with countable support. Suppose $p \in(0,1)$ to fulfill

$$
\inf _{z \in \mathbb{R}^{s}}\left|F_{\mu}(z)-p\right|>0
$$

Then the constraint function $F_{\mu}(h(\cdot))-p$ is metrically regular at all feasible $x^{0}$ w.r.t. $C$, whereas it is not growing w.r.t. $C$ at all $x^{0}$ such that $F_{\mu}$ is not continuous at $h\left(x^{0}\right)$.

\subsection{Separate Constraint Qualifications}

While metric regularity of the probabilistic constraint (7) has been characterized in terms of the composite function $\Phi_{\mu} \circ h$ so far, we now want to formulate separate constraint qualifications for the two single functions that are easier to verify and to interpret. With the constraint functions $\Phi_{\mu}$ and $h$ from the definition of the probabilistic constraint in (7) we associate the following two multifunctions $\Gamma_{1}: \mathbb{R}_{\stackrel{s}{\rightarrow}}^{\rightarrow} \mathbb{R}^{d}$ and $\Gamma_{2}: \mathbb{R}_{\stackrel{m}{m} \rightarrow}^{\rightarrow} \mathbb{R}^{s}$ via

$$
\Gamma_{1}(z)=p-\Phi_{\mu}(z)+\mathbb{R}_{+}^{d} \quad \text { and } \quad \Gamma_{2}(x)= \begin{cases}h(x) & x \in C \\ \emptyset & \text { else }\end{cases}
$$

Then, their composition is $\Gamma=\Gamma_{1} \circ \Gamma_{2}: \mathbb{R}^{m} \rightarrow \mathbb{R}^{d}$ with

$$
\Gamma(x)= \begin{cases}p-\Phi_{\mu}(h(x))+\mathbb{R}_{+}^{d} & x \in C \\ \emptyset & \text { else }\end{cases}
$$

Proposition 3.15 In (7), assume $h$ to be continuous and consider some feasible point $\bar{x} \in M$. Then the two constraint qualifications

$$
\begin{aligned}
\operatorname{Ker} D_{a}^{*} \Gamma_{1}(h(\bar{x}), 0) & =\{0\} \\
\operatorname{Ker} D_{a}^{*} \Gamma_{2}(\bar{x}, h(\bar{x})) \cap \mathbb{R}_{-}^{s} & =\{0\}
\end{aligned}
$$

imply $\operatorname{Ker} D_{a}^{*} \Gamma(\bar{x}, 0)=\{0\}$.

Proof:

All of the three multifunctions $\Gamma_{2}, \Gamma_{1}$ and $\Gamma$ have a closed graph (due to the closedness of $C$, continuity of $h$ and upper semicontinuity of $\left.\Phi_{\mu}\right)$. Let us assume for a moment that the application of Theorem 3.2 is justified. Then the relation $0 \in D_{a}^{*} \Gamma(\bar{x}, 0)\left(z^{*}\right)$ (for arbitrary $z^{*}$ ) along with the fact that $\Gamma_{2}$ is single-valued $\left(\Gamma_{2}(\bar{x})=h(\bar{x})\right)$ yield the existence of some $y^{*} \in \mathbb{R}^{s}$ such that

$$
y^{*} \in D_{a}^{*} \Gamma_{1}(h(\bar{x}), 0)\left(z^{*}\right) \quad \text { and } \quad 0 \in D_{a}^{*} \Gamma_{2}(\bar{x}, h(\bar{x}))\left(y^{*}\right) .
$$

¿From Proposition 3.8 we know that $\operatorname{Im} D_{a}^{*} \Gamma_{1}(h(\bar{x}), 0) \subseteq \mathbb{R}_{-}^{s}$. This leads to

$$
y^{*} \in \operatorname{Ker} D_{a}^{*} \Gamma_{2}(\bar{x}, h(\bar{x})) \cap \mathbb{R}_{-}^{s}=\{0\}
$$

by (17) and to $z^{*} \in \operatorname{Ker} D_{a}^{*} \Gamma_{1}(h(\bar{x}), 0)=\{0\}$ by (16). Consequently, $D_{a}^{*} \Gamma(\bar{x}, 0)=\{0\}$, as desired. 
To check the assumptions of Theorem 3.2 first note that the multifunction $M(x, z)=$ $\Gamma_{2}(x) \cap \Gamma_{1}^{-1}(z)$ fulfills either $M(x, z)=\emptyset$ or $M(x, z)=\{h(x)\}$, so it is locally bounded by continuity of $h$. In particular, $M(\bar{x}, \bar{z})=\{h(\bar{x})\}$, and again from Proposition 3.8 and (17) we have

$$
D_{a}^{*} \Gamma_{1}(h(\bar{x}), 0)(0) \cap \operatorname{Ker} D_{a}^{*} \Gamma_{2}(\bar{x}, h(\bar{x})) \subseteq \mathbb{R}_{-}^{s} \cap \operatorname{Ker} D_{a}^{*} \Gamma_{2}(\bar{x}, h(\bar{x}))=\{0\} .
$$

The result of this proposition can now be restated in terms of the ingredients of the probabilistic constraint (7) itself.

Theorem 3.16 The constraint function $\Phi_{\mu}(h(\cdot))-p$ in $(\boldsymbol{\eta})$ is metrically regular at some feasible point $\bar{x} \in M$ w.r.t. $C$ if the following two conditions are fulfilled:

(i) The function $\Phi_{\mu}(\cdot)-p$ is metrically regular at $h(\bar{x})$ in the constraint $\Phi_{\mu}(z) \geq p$.

(ii) $h$ is continuous, $N_{a}(\operatorname{Gph} h ;(\bar{x}, h(\bar{x}))) \cap\left(-N_{a}(C ; \bar{x}) \times\{0\}\right)=\{0\}$ and

$$
D_{a}^{*} h(\bar{x}, h(\bar{x}))\left(y^{*}\right) \cap-N_{a}(C ; \bar{x})=\emptyset \quad \forall y^{*} \in \mathbb{R}_{-}^{s} \backslash\{0\}
$$

\section{Proof:}

Obviously, condition (i) is equivalent to (16) by Theorem 3.1. Concerning (ii) one has Gph $\Gamma_{2}=$ Gph $h \cap\left(C \times \mathbb{R}^{s}\right)$ for the multifunction $\Gamma_{2}$ introduced above. The first part of (ii) corresponds to the assumption of Lemma 3.3 (with $S_{1}=\mathrm{Gph} h$ and $S_{2}=C \times \mathbb{R}^{s}$ ), so the lemma yields

$$
N_{a}\left(\operatorname{Gph} \Gamma_{2} ;(\bar{x}, h(\bar{x}))\right) \subseteq N_{a}(\operatorname{Gph} h ;(\bar{x}, h(\bar{x})))+N_{a}(C ; \bar{x}) \times\{0\}
$$

Choose any $y^{*} \in \operatorname{Ker} D_{a}^{*} \Gamma_{2}(\bar{x}, h(\bar{x})) \cap \mathbb{R}_{-}^{s}$. In particular, $\left(0,-y^{*}\right) \in N_{a}\left(\operatorname{Gph} \Gamma_{2} ;(\bar{x}, h(\bar{x}))\right)$ and we have $\left(0,-y^{*}\right)=(\xi, a)+(\pi, 0)$ according to the decomposition just stated. Then $\xi=-\pi \in$ $-N_{a}(C ; \bar{x})$ and $\left(\xi,-y^{*}\right)=(\xi, a) \in N_{a}(\operatorname{Gph} h ;(\bar{x}, h(\bar{x})))$. It follows $\xi \in D_{a}^{*} h(\bar{x}, h(\bar{x}))\left(y^{*}\right) \cap$ $-N_{a}(C ; \bar{x})$, hence $y^{*}=0$ due to the second part in (ii) and to $y^{*} \in \mathbb{R}_{-}^{s}$. However, this is (17), so Proposition 3.15 guarantees $\operatorname{Ker} D_{a}^{*} \Gamma(\bar{x}, 0)=\{0\}$ and, Theorem 3.1 implies metric regularity of $\Phi_{\mu}(h(\cdot))-p$ at $\bar{x}$ w.r.t $C$.

Theorem 3.16 offers the possibility to check properties of the measure $\mu$ and of the function $h$ in (7) separately. Yet the conditions imposed are rather abstract. In the following we develop criteria that are better to verify. First we turn to condition (i) and try to reformulate it in terms of assumptions concerning the density of the measure $\mu$. If $\mu$ has a density, then, denoting

$$
y=\left(y_{1}^{1}, \ldots, y_{1}^{s_{1}}, \ldots, y_{d}^{1}, \ldots, y_{d}^{s_{d}}\right) \quad\left(y \in \mathbb{R}^{s} ; s=s_{1}+\cdots+s_{d}\right)
$$

one recognizes that the components of $\Phi_{\mu}$ may be written as

$$
\Phi_{\mu}^{j}(y)=\int_{-\infty}^{\infty} \cdots \int_{-\infty}^{\infty} \int_{-\infty}^{y_{j}^{1}} \cdots \int_{-\infty}^{y_{j}^{s_{j}}} \int_{-\infty}^{\infty} \cdots \int_{-\infty}^{\infty} f_{\mu}(y) d y_{d}^{s_{d}} \cdots d y_{j+1}^{1} d y_{j}^{s_{j}} \cdots d y_{j}^{1} d y_{j-1}^{s_{j-1}} \cdots d y_{1}^{1}
$$


Next we introduce the set where this density is locally bounded below by a positive number:

$$
\mathcal{D}^{+}=\left\{y \in \mathbb{R}^{s} \mid \exists \varepsilon>0 \forall \tilde{y} \in B_{\varepsilon}(y): f_{\mu}(\tilde{y}) \geq \varepsilon\right\} .
$$

For continuous $f_{\mu}$, of course, this set reduces to $\mathcal{D}^{+}=\left\{y \in \mathbb{R}^{s} \mid f_{\mu}(y)>0\right\}$. Finally, for any subset $I \subseteq\{1, \ldots, d\}$ put

$$
\Omega^{I}=C_{1} \times \cdots \times C_{d}, \quad \text { where } C_{i}= \begin{cases}\mathbb{R}^{s_{i}} & i \notin I \\ \partial \mathbb{R}_{-}^{s_{i}} & i \in I\end{cases}
$$

The following theorem provides a density condition guaranteeing sufficient growth of $\Phi_{\mu}$ to arrive at the desired property of metric regularity.

Theorem 3.17 For $\bar{x} \in M$ in (7), denote the set of active indices by $I(\bar{x})=\{i \in\{1, \ldots, d\} \mid$ $\left.\Phi_{\mu}^{i}(h(\bar{x}))=p^{i}\right\}$. If $\mu$ has a density and $\left(h(\bar{x})+\Omega^{I(\bar{x})}\right) \cap \mathcal{D}^{+} \neq \emptyset$, then condition (i) of Theorem 3.16 is satisfied.

\section{Proof:}

By assumption, there exists some $\bar{y} \in \mathcal{D}^{+}$such that for all $j \in I(\bar{x})$

$$
\bar{y}_{j}^{k} \leq[h(\bar{x})]_{j}^{k} k=1, \ldots, s_{j} \quad \text { and } \quad \exists k(j) \in\left\{1, \ldots, s_{j}\right\}: \bar{y}_{j}^{k(j)}=[h(\bar{x})]_{j}^{k(j)}
$$

Here, lower and upper indices refer to the partition of vectors in $\mathbb{I}^{s}=\mathbb{R}^{s_{1}} \times \cdots \times \mathbb{R}^{s_{d}}$ introduced above. By definition, one has $f_{\mu}(y) \geq \varepsilon$ for all $y \in B_{\varepsilon}(\bar{y})$. Choose any $z \in$ $B_{\varepsilon / 2}(h(\bar{x}))$. Without loss of generality we consider the balls with respect to the maximum norm $\|\cdot\|_{\infty}$. As a consequence, we have for all indices $j \in I(\bar{x})$

$$
z_{j}^{k} \geq \bar{y}_{j}^{k}-\varepsilon / 2 \quad k=1, \ldots, s_{j} .
$$

Next define some vector $e \in \mathbb{R}^{s}$ via

$$
e_{j}^{k}= \begin{cases}1 & j \in I(\bar{x}) \text { and } k=k(j) \\ 0 & \text { else }\end{cases}
$$

and put $z(t)=z+$ te for $t \in(0, \varepsilon / 2)$. Clearly, for all indices $j \in I(\bar{x})$ it holds

$$
[z(t)]_{j}^{k(j)}=z_{j}^{k(j)}+t \quad \text { and } \quad[z(t)]_{j}^{k}=z_{j}^{k} \quad \text { if } k \neq k(j)
$$

In particular, $\|z(t)-z\|_{\infty}=t$ and for $c \in\left[z_{j}^{k(j)}, z_{j}^{k(j)}+t\right]$ one has

$$
\left|c-\bar{y}_{j}^{k(j)}\right| \leq\left|c-z_{j}^{k(j)}\right|+\left|z_{j}^{k(j)}-[h(\bar{x})]_{j}^{k(j)}\right| \leq \varepsilon / 2+\varepsilon / 2=\varepsilon .
$$

Now, the following estimation can be made for the active indices $j \in I(\bar{x})$ :

$$
\Phi_{\mu}^{j}(z(t))-\Phi_{\mu}^{j}(z)=\int_{-\infty}^{\infty} \cdots \int_{-\infty}^{\infty} \int_{-\infty}^{[z(t)]_{j}^{1}} \cdots \int_{-\infty}^{[z(t)]_{j}^{k(j)}} \cdots \int_{-\infty}^{[z(t)]_{j}^{s_{j}}} \int_{-\infty}^{\infty} \cdots \int_{-\infty}^{\infty} f_{\mu}(y) d y
$$




$$
\begin{aligned}
& -\int_{-\infty}^{\infty} \cdots \int_{-\infty}^{\infty} \int_{-\infty}^{z_{j}^{1}} \cdots \int_{-\infty}^{z_{j}^{k(j)}} \cdots \int_{-\infty}^{z_{j}^{s_{j}}} \int_{-\infty}^{\infty} \cdots \int_{-\infty}^{\infty} f_{\mu}(y) d y \\
& =\int_{-\infty}^{\infty} \cdots \int_{-\infty}^{\infty} \int_{-\infty}^{z_{j}^{1}} \cdots \int_{z_{j}^{k(j)}}^{z_{j}^{k(j)}+t} \cdots \int_{-\infty}^{z_{j}^{s_{j}}} \int_{-\infty}^{\infty} \cdots \int_{-\infty}^{\infty} f_{\mu}(y) d y \\
& \geq \int_{\bar{y}_{1}^{1}-\varepsilon}^{\bar{y}_{1}^{1}} \cdots \int_{\bar{y}_{j-1}^{s_{j}-1}-\varepsilon}^{\bar{y}_{j-1}^{s_{j-1}}} \int_{\bar{y}_{j}^{1}-\varepsilon}^{\bar{y}_{j}^{1}-\varepsilon / 2} \cdots \int_{z_{j}^{k(j)}}^{z_{j}^{k(j)}+t} \cdots \int_{\bar{y}_{j}^{s_{j}}-\varepsilon}^{\bar{y}_{j}^{s_{j}}-\varepsilon / 2} \int_{\bar{y}_{j+1}^{1}-\varepsilon}^{\bar{y}_{j+1}^{1}} \cdots \int_{\bar{y}_{d}^{s} d-\varepsilon}^{\bar{y}_{d}^{s_{d}}} \varepsilon d y \\
& =\varepsilon^{s-s_{j}} \cdot(\varepsilon / 2)^{s_{j}-1} \cdot \varepsilon \cdot\|z(t)-z\|_{\infty}
\end{aligned}
$$

But, having in mind, that $\Phi_{\mu}$ is continuous due to the assumption that $\mu$ possesses a density, the above estimation results in $\Phi_{\mu}(\cdot)-p$ being growing at $h(\bar{x})$ (w.r.t. $\mathbb{R}^{s}$ ) in the sense of Definition 3.4 (put $\rho=(\varepsilon / 2)^{s}, \eta=\varepsilon / 2$ and recall that the above estimation is valid for all $t \in(0, \varepsilon / 2)$ ). According to Lemma $3.5 \Phi_{\mu}(\cdot)-p$ (considered with the $\geq 0$ constraint) is metrically regular at $h(\bar{x})$. This is condition (i) of Theorem 3.16.

Since, by definition, $0 \in \Omega^{I(\bar{x})}$ for whatever index set $I(\bar{x})$, one concludes

Corollary 3.18 If $h(\bar{x}) \in \mathcal{D}^{+}$, then condition (i) of Theorem 3.16 is satisfied.

This density condition $h(\bar{x}) \in \mathcal{D}^{+}$was used in [43] (Lemma 2.1) in order to derive a corresponding stability result for a specific probabilistic constraint $(d=1$ and $h$ linear in $(7))$. For continuous densities one simply would have to require $f_{\mu}(h(\bar{x}))>0$. Note, however, that this relation is far from being necessary in order to ensure condition (i) of Theorem 3.16, as can be seen from the following example:

Example 3.19 In (7), we take $d=1, s=m=2, h(x)=x, p=0.5, C=\mathbb{R}^{2}$. In particular, $\Phi_{\mu}$ coincides with the distribution function of the measure $\mu$, which we assume to be induced by the following density on $\mathbb{R}^{2}$ :

$$
f_{\mu}(y)= \begin{cases}a & y \in B_{1}(0) \\ (2-\|y\|) a & y \in B_{2}(0) \backslash B_{1}(0) \\ 0 & y \in \mathbb{R}^{2} \backslash B_{2}(0)\end{cases}
$$

where the balls of the corresponding distances refer to the Euclidean norm and the number $a>0$ is suitably chosen to guarantee $\int_{\mathbb{R}^{2}} f_{\mu}(y) d y=1$. Obviously, $f_{\mu}$ is continuous and $\mathcal{D}^{+}=\operatorname{int} B_{2}(0)$. For $\bar{x}=(0,3)$ we deduce from the symmetry of $f_{\mu}$ around the origin that

$$
\Phi_{\mu}(h(\bar{x}))=\Phi_{\mu}((0,3))=0.5=p,
$$

hence, we have the binding case $I(\bar{x})=\{1\}$. Of course, $f_{\mu}(h(\bar{x}))=f_{\mu}((0,3))=0$, so the strong condition of Corollary 3.18 does not apply. Nevertheless, one may derive condition (i) of Theorem 3.16 because $\left[(0,3)+\partial \mathbb{R}_{-}^{2}\right] \cap \mathcal{D}^{+} \neq \emptyset$ (take, for instance $(0,-3) \in \partial \mathbb{R}_{-}^{2}$ ), hence, the weaker condition in Theorem 3.17 is satisfied. 
Frequently, the property of metric regularity is required at points that are not given explicitly, e.g. the set of local minimizers. Therefore, it might sometimes be useful to know conditions under which metric regularity holds everywhere. For instance, as a part of this question, one could ask when condition (i) of Theorem 3.16 is satisfied everywhere, i.e., $\Phi_{\mu}(\cdot)-p$ is metrically regular at all $h(\bar{x})$ with $\bar{x} \in M$. Using Corollary 3.18 one gets an immediate criterion for such a global behaviour, namely $\mathcal{D}^{+}=\mathbb{R}^{s}$, which is fulfilled for some of the conventional distributions (like multivariate normal). The situation becomes more interesting for densities whose support is not all of $R^{s}$. To investigate this problem in more detail we introduce the following definition:

Definition $3.20 A$ subset $Q \subseteq \mathbb{R}^{n}$ is called an infinity path in $\mathbb{R}^{n}$ if there exists some continuous function $\pi: \mathbb{R} \rightarrow \mathbb{R}^{n}$ such that $\operatorname{Im} \pi=Q$ and

$$
\lim _{t \rightarrow-\infty} \max _{i=1, \ldots, n} \pi_{i}(t)=-\infty, \quad \lim _{t \rightarrow \infty} \max _{i=1, \ldots, n} \pi_{i}(t)=\infty
$$

Roughly speaking, one part of $Q$ must tend to $-\infty$ with all its coordinates simultaneously, while for the other part it suffices that at least one coordinate tends to $+\infty$. Of course, any infinity path is a connected subset of $\mathbb{R}^{n}$. This concept allows an appropriate characterization in the case of $\Phi_{\mu}$ having only one component, i.e., $d=1$.

Theorem 3.21 If $d=1, \mu$ has a density and $\mathcal{D}^{+}$contains an infinity path $Q$ in $R^{s}$, then condition (i) in Theorem 3.16 holds globally, i.e., $\Phi_{\mu}(\cdot)-p$ is metrically regular at $h(\bar{x})$ for all $\bar{x} \in M$.

\section{Proof:}

Consider any $\bar{x} \in M$ and put $z=h(\bar{x})$. With reference to Definition 3.20 there exist $t_{1}, t_{2} \in \mathbb{R}$, such that

$$
\max _{i=1, \ldots, s} \pi_{i}\left(t_{1}\right)<\min _{i=1, \ldots, s} z_{i}, \quad \max _{i=1, \ldots, s} \pi_{i}\left(t_{2}\right)>\max _{i=1, \ldots, s} z_{i}
$$

Hence, for $q_{1}=\pi\left(t_{1}\right), q_{2}=\pi\left(t_{2}\right)$ one has $q_{1} \in Q \cap \operatorname{int}\left(z+\mathbb{R}_{-}^{s}\right)$ and $q_{2} \in Q \cap\left(\mathbb{R}^{s} \backslash\left(z+\mathbb{R}_{-}^{s}\right)\right)$. Now

$$
\mathbb{R}^{s}=\left[\operatorname{int}\left(z+\mathbb{R}_{-}^{s}\right)\right] \cup\left[\mathbb{R}^{s} \backslash\left(z+\mathbb{R}_{-}^{s}\right)\right] \cup\left[z+\partial \mathbb{R}_{-}^{s}\right]
$$

is a disjoint decomposition of $\mathbb{R}^{s}$, where the first two sets are open. Therefore $Q \cap\left(z+\partial \mathbb{R}_{-}^{s}\right) \neq \emptyset$ because otherwise

$$
Q=\left[Q \cap \operatorname{int}\left(z+\mathbb{R}_{-}^{s}\right)\right] \cup\left[Q \cap\left(\mathbb{R}^{s} \backslash\left(z+\mathbb{R}_{-}^{s}\right)\right)\right]
$$

would be a decomposition of $Q$ into two open, disjoint and nonempty subsets in contradiction to the connectedness of $Q$. Taking account of $Q \subseteq \mathcal{D}^{+}$, we arrive at

$$
\emptyset \neq \mathcal{D}^{+} \cap\left(z+\partial \mathbb{I}_{-}^{s}\right) \subseteq \mathcal{D}^{+} \cap\left(h(\bar{x})+\Omega^{I(\bar{x})}\right)
$$

Since $\bar{x} \in M$ was arbitrary, the assertion follows from Theorem 3.17 .

It is noted here, that the assertion of the theorem is not restricted to the fixed probability level $p$, in fact, this value does not enter the proof at any point. Consequently, under the indicated assumptions, $\Phi_{\mu}(\cdot)-p^{\prime}$ is metrically regular at $h(\bar{x})$ not only for all $\bar{x} \in M$ but even for all $p^{\prime} \in(0,1)$. The following example shall illustrate the meaning of Theorem 3.21. 
Example 3.22 Adopt the setting of Example 3.19, but with the density on $\mathbb{R}^{2}$ replaced by

$$
f_{\mu}(y)= \begin{cases}a e^{-y_{1}^{2} / 2} & \text { if } y_{1} \geq 0,\left|y_{2}\right|<1 / 2 \quad \text { or } \quad y_{1}<0,\left|y_{2}-y_{1}\right|<1 / 2 \\ 0 & \text { else }\end{cases}
$$

(a such that $\int f_{\mu}(y) d y=1$ ). Obviously, here the set $\mathcal{D}^{+}$coincides with the one which the first line in the definition of $f_{\mu}$ relates to, so $\mathcal{D}^{+} \neq \mathbb{I}^{2}$. Nevertheless, condition (i) of Theorem 3.16 is satisfied for all (!) $\bar{x} \in \mathbb{R}^{2}$. In fact, the continuous function $\pi: \mathbb{R} \rightarrow \mathbb{R}^{2}$ defined by $\pi(t)=(t, t)$ if $t \leq 0$ and $\pi(t)=(t, 0)$ if $t>0$ generates an infinity path $Q=\operatorname{Im} \pi$ that is contained in $\mathcal{D}^{+}$, so Theorem 3.21 applies.

Now, reflect the density w.r.t. the origin, i.e., take $f_{\mu^{\prime}}(y)=f_{\mu}(-y)$. Then, the set $\mathcal{D}^{+^{\prime}}$ does not contain any infinity path. For instance, the canonical candidate $Q^{\prime} \subseteq \mathcal{D}^{+^{\prime}}$, which is defined by $Q^{\prime}=\operatorname{Im} \pi^{\prime}$, where $\pi^{\prime}(t)=(t, 0)$ for $t \leq 0$ and $\pi^{\prime}(t)=(t, t)$ for $t>0$, fails to satisfy the first limiting condition in Definition 3.20 (while the second one holds true).

Now we turn to the second constraint qualification in Theorem 3.16. As will be seen below, this can be viewed as some kind of Mangasarian-Fromovitz Constraint Qualification for continuous inequality constraints. The first part of this condition (relating the approximate normal cones of the Graph of $h$ and of the set $C$ ) is always fulfilled, for instance, if $C=\mathbb{R}^{m}$ or if $h$ is locally Lipschitzian. In order to gain more insight, we consider the cases of locally Lipschitzian or even $C^{1}$ - mappings $h$.

Proposition 3.23 If $h$ is locally Lipschitzian in (7), then condition (ii) of Theorem 3.16 reduces to

$$
\partial_{a}\left\langle y^{*}, h\right\rangle(\bar{x}) \cap-N_{a}(C ; \bar{x})=\emptyset \quad \forall y^{*} \in \mathbb{R}_{-}^{s} \backslash\{0\} .
$$

If $h \in \mathcal{C}^{1}\left(\mathbb{R}^{m}, \mathbb{R}^{s}\right)$ with Jacobian $D h$, then the corresponding relation reads

$$
[D h(\bar{x})]^{T} y^{*} \notin N_{a}(C ; \bar{x}) \quad \forall y^{*} \in \mathbb{R}_{+}^{s} \backslash\{0\}
$$

\section{Proof:}

For locally Lipschitzian $h$ the first part of condition (ii) in Theorem 3.16 is automatically fulfilled. In fact, if $k$ is a Lipschitz modulus of $h$ near $\bar{x}$, then $\left\|a^{*}\right\| \leq k\left\|b^{*}\right\|$ for all $\left(a^{*}, b^{*}\right) \in$ $T^{0}(\operatorname{Gph} h ;(x, h(x)))$ and all $x$ near $\bar{x}$ (compare [20], Lemma 3.8). Now, the same relation must hold true for all $\left(a^{*}, b^{*}\right) \in N_{a}\left((\operatorname{Gph} h ;(\bar{x}, h(\bar{x})))\right.$ too. In particular, $b^{*}=0$ implies $a^{*}=0$.

Finally, the second part of condition (ii) is nothing else but (18) as a consequence of (9). Now (19) follows from the fact that the approximate subdifferential and the usual derivative coincide in the $\mathcal{C}^{1}$ - case.

In case $C=\mathbb{R}^{m}$ (i.e., $N_{a}(C ; \bar{x})=\{0\}$ ), Gordan's theorem shows the equivalence of (19) with the condition

$$
\exists \xi \in \mathbb{R}^{m}: \quad \nabla h_{i}(\bar{x}) \cdot \xi>0 i=1, \ldots, s,
$$

where now, in contrast to the derivations above, we return to the conventional labelling of the components of $h$. Restricting this relation to the active indices only (which have no meaning for $h$ in our present context) this would be the well-known Mangasarian-Fromovitz Constraint 
Qualification (in the absence of equations). Replacing the sets in (18) by the corresponding (bigger) concepts of Clarke's subdifferential calculus, one gets the stronger requirement

$$
\partial_{c}\left\langle y^{*}, h\right\rangle(\bar{x}) \cap N_{c}(C ; \bar{x})=\emptyset \quad \forall y^{*} \in \mathbb{R}_{+}^{s} \backslash\{0\},
$$

which is closely related to well-known constraint qualifications in the locally Lipschitzian setting (e.g. [40], [9], [3], [21]). However, let us emphasize once more that, in (7), the mapping $h$ does not appear itself as a constraint, but as the inner part of a composite constraint. In particular, there is no active index set to be considered. Furthermore, the application of (18) according to Mordukhovich's calculus promises advantages over (20) for certain classes of mappings, like nonsmooth convex ones, the most trivial example being $h(x)=|x|, C=\mathbb{R}$, where (18) is satisfied at zero due to $\partial_{a}(-|\cdot|)(0)=\{-1,1\}$ while (20) fails to hold.

Similar to the considerations with respect to condition (i) in Theorem 3.16 one may ask under which circumstances condition (ii) of the same theorem holds globally, i.e., for all $\bar{x} \in M$. An answer may be deduced from the following corollary to Proposition 3.23:

Corollary 3.24 In (7), let all components of $h$ be concave and the set $C$ be convex. If, for $\bar{x} \in M$, there exists some $x^{*} \in C$ such that $h\left(x^{*}\right)>h(\bar{x})$ (componentwise), then condition (ii) of Theorem 3.16 is satisfied.

\section{Proof:}

Due to concavity, $h$ is locally Lipschitzian, so we have to check (18). If this relation does not hold, then there exist some $y^{*} \in \mathbb{R}_{+}^{s} \backslash\{0\}$ and $\xi \in \mathbb{R}^{m}$ such that $\xi \in \partial\left\langle y^{*},-h\right\rangle(\bar{x}) \cap-N(C ; \bar{x})$ (note that $\left\langle y^{*},-h\right\rangle$ is convex and that $\partial_{a}$ and $N_{a}$ coincide with the subdifferential $\partial$ and the normal cone $N$ of convex analysis). Since both $\bar{x}$ and $x^{*}$ belong to the convex set $C$, we derive $\left\langle\xi, x^{*}-\bar{x}\right\rangle \geq 0$. On the other hand, by the sum rule of the convex subdifferential, there are $\xi_{i} \in \partial\left(-h_{i}\right)(\bar{x})$ with $\xi=\sum_{i=1}^{s} y_{i}^{*} \xi_{i}$. In particular, by the definition of the convex subdifferential, one has $\left\langle\xi_{i}, x^{*}-\bar{x}\right\rangle \leq h_{i}(\bar{x})-h_{i}\left(x^{*}\right)$. Summarizing, one obtains the contradiction

$$
0 \leq\left\langle\xi, x^{*}-\bar{x}\right\rangle=\sum_{i=1}^{s} y_{i}^{*}\left\langle\xi_{i}, x^{*}-\bar{x}\right\rangle \leq \sum_{i=1}^{s} y_{i}^{*}\left(h_{i}(\bar{x})-h_{i}\left(x^{*}\right)\right)<0
$$

from the strict inequality in the assumption.

The corollary corrects an error in [43] Lemma 2.1., where, in the context of linear mappings $h$ and convex sets $C$, the existence of some $x^{*} \in C$ with $h\left(x^{*}\right) \geq h(\bar{x})$ was required instead of the strict inequality.

Now, the desired global property may be formulated as follows: If, in (7), $h$ is concave (e.g. linear) and $C$ is convex, then condition (ii) of Theorem 3.16 is fulfilled at all $\bar{x} \in M$ except for the pareto optimal points of the vector optimization problem

$$
\max \left\{h_{i}(x) \mid x \in C, i=1, \ldots, s\right\} .
$$

At the end of this section we reexamine Example 3.13 using the tools related to Theorem 3.16. In contrast to the previously given verification of metric regularity by means of the composite function $\Phi_{\mu} \circ h$, the corresponding result shall be obtained now via separate considerations of the measure and the function $h$. 
Example 3.25 (Example 3.13 revisited) Due to $N_{a}(C ;(0,0))=\left\{\left(\xi_{1}, \xi_{2}\right) \in \mathbb{R}^{2} \mid \xi_{1} \leq 0\right\}$ one has

$$
[D h(0,0)]^{T} y^{*}=\left(y^{*}, y^{*}\right)^{T} \notin N_{a}(C ;(0,0)) \quad \forall y^{*}>0 .
$$

Consequently, (19) applies. On the other hand $\mathcal{D}^{+}=(-0.5,0.5)$ for the given unifrom distribution over $[-0.5,0.5]$. So $h(0,0)=0 \in \mathcal{D}^{+}$and we are in the situation of Corollary 3.18. Summarizing, both conditions of Theorem 3.16 are satisfied and the desired metric regularity result follows.

\section{Quadratic growth condition and quantitative stability}

In order to obtain quantitative stability results for solution sets, a certain growth condition for the objective function in a neighbourhood of the optimal set has to be verified. This is studied next for more specific (convex) stochastic programs with one joint probabilistic constraint and polyhedral deterministic constraints. More precisely, we consider the problem

$$
\mathbf{P}(\mu) \quad \min \left\{g(x) \mid x \in C, F_{\mu}(A x) \geq p\right\},
$$

where $g: \mathbb{R}^{m} \rightarrow \mathbb{R}$ is convex quadratic, $C \subseteq \mathbb{R}^{m}$ is convex polyhedral, $A$ is an $(s, m)$ matrix, $p \in(0,1)$ and $F_{\mu}$ is the distribution function of a probability measure $\mu \in \mathcal{P}\left(\mathbb{R}^{s}\right)$, which is assumed to be $r$-concave for some $r \in(-\infty, 0)$. Due to the $r$-concavity of $\mu, \mathbf{P}(\mu)$ represents a convex program. In the following, $\Psi(\mu)$ refers to the set of (global) solutions to (21) and, as in Section 2, $\Psi_{V}(\nu)$ denotes the localized solution set to $\mathbf{P}(\nu)$, where $\nu \in \mathcal{P}\left(\mathbb{R}^{s}\right)$ is a perturbation of $\mu$ and $V \subseteq \mathbb{R}^{m}$ an open neighbourhood of $\Psi(\mu)$.

In the first step of our analysis a reduction argument is used to decompose the original problem $\mathbf{P}(\mu)$ into two auxiliary problems. The first one is a stochastic program under probabilistic constraints, again with decisions taken in $R^{s}$, whereas the second one represents a parametric quadratic program with polyhedral constraints. The reduction argument also provides insight into the structure of the solution set $\Psi(\mu)$. A similar argument was already used in a different context in the proof of Theorem 2.4 in [45].

Proposition 4.1 In addition to the general assumptions, let $\nu \in \mathcal{P}\left(\mathbb{R}^{s}\right)$ and suppose the closure $\mathrm{cl} V$ of $V \subseteq \mathbb{R}^{m}$ to be a polytope. Then we have

$$
\varphi_{V}(\nu)=\inf \left\{\pi_{V}(y) \mid y \in A\left(C_{V}\right), F_{\nu}(y) \geq p\right\} \quad \text { and } \quad \Psi_{V}(\nu)=\sigma_{V}\left(Y_{V}(\nu)\right),
$$

where

$$
\begin{aligned}
Y_{V}(\nu) & =\operatorname{argmin}\left\{\pi_{V}(y) \mid y \in A\left(C_{V}\right), F_{\nu}(y) \geq p\right\} \\
C_{V} & =C \cap \operatorname{cl} V \\
\pi_{V}(y) & =\inf \left\{g(x) \mid A x=y, x \in C_{V}\right\} \\
\sigma_{V}(y) & =\operatorname{argmin}\left\{g(x) \mid A x=y, x \in C_{V}\right\}\left(y \in A\left(C_{V}\right)\right) .
\end{aligned}
$$

Here, $\pi_{V}$ is convex on $A\left(C_{V}\right), \sigma_{V}$ is Hausdorff Lipschitzian on $A\left(C_{V}\right)$ and there exists an $\eta>0$ such that

$$
g(x) \geq \pi_{V}(A x)+\eta d\left(x, \sigma_{V}(A x)\right)^{2} \quad \forall x \in C_{V} .
$$




\section{Proof:}

Since the constraint set $\left\{x \in C_{V} \mid F_{\nu}(A x) \geq p\right\}$ is compact, $\Psi_{V}(\nu)$ is nonempty. Let $x \in \Psi_{V}(\nu)$. Then $x \in C_{V}, F_{\nu}(A x) \geq p$, and

$$
\varphi_{V}(\nu)=g(x) \geq \pi_{V}(A x) \geq \inf \left\{\pi_{V}(y) \mid y \in A\left(C_{V}\right), F_{\nu}(y) \geq p\right\} .
$$

Conversely, let $y \in A\left(C_{V}\right)$ with $F_{\nu}(y) \geq p$. Then there exists an $x \in \sigma_{V}(y)$ with $\pi_{V}(y)=$ $g(x) \geq \varphi_{V}(\nu)$. Hence

$$
\varphi_{V}(\nu)=\inf \left\{\pi_{V}(y) \mid y \in A\left(C_{V}\right), F_{\nu}(y) \geq p\right\} \quad \text { and } \quad g(x)=\pi_{V}(A x) \quad \forall x \in \Psi_{V}(\nu) .
$$

This implies $\Psi_{V}(\nu)=\sigma_{V}\left(Y_{V}(\nu)\right)$. The convexity of $\pi_{V}$ is immediate and the Lipschitz property of $\sigma_{V}$ is shown in [27], Theorem 4.2. Finally, the proof of the last statement in the lemma is based on Hoffman's theorem. It is omitted here for the sake of brevity.

The preceding result enables us first to study the growth behaviour of the objective function in the auxiliary problem

$$
\min \left\{\pi_{V}(y) \mid y \in A\left(C_{V}\right), F_{\nu}(y) \geq p\right\},
$$

where $V$ is some suitably chosen subset of $\mathbb{R}^{m}$. In a second step, the formula for $\Psi_{V}$ in the above proposition and the properties of $\sigma_{V}$ may be exploited. This two-stage procedure forms the basis of the proof of the following results.

Theorem 4.2 In addition to the general assumptions in this section, suppose that

(i) $\Psi(\mu)$ is nonempty and bounded;

(ii) $\Psi(\mu) \cap \operatorname{argmin}\{g(x) \mid x \in C\}=\emptyset$;

(iii) $\exists \bar{x} \in C: F_{\mu}(A \bar{x})>p$ (Slater condition);

(iv) $F_{\mu}^{r}$ is strongly convex on some open convex neighbourhood $U$ of $A(\Psi(\mu))$, where $r \in$ $(-\infty, 0)$ is chosen such that $\mu$ is r-concave.

Then the following quadratic growth condition is satisfied:

$$
\exists c>0 \exists V \supseteq \Psi(\mu)(V \text { open }): \quad g(x) \geq \varphi(\mu)+c d(x, \Psi(\mu))^{2} \quad \forall x \in C \cap V, F_{\mu}(A x) \geq p .
$$

\section{Proof:}

Let $V_{0} \subseteq \mathbb{R}^{m}$ be an open convex set such that $\Psi(\mu) \subseteq V_{0}$ and $A\left(V_{0}\right) \subseteq U$. For each $x \in \Psi(\mu)$ select $\varepsilon(x)>0$ such that the closed ball (w.r.t. the norm $\left.\|\cdot\|_{\infty}\right) B_{\infty}(x, \varepsilon(x)$ ) around $x$ with radius $\varepsilon(x)$ is contained in $V_{0}$. Since $\Psi(\mu)$ is compact, a finite number of these balls cover $\Psi(\mu)$. The closed convex hull $\bar{V}$ of their union is a polyhedron with $\Psi(\mu) \subseteq V \subset \bar{V} \subseteq V_{0}$, where $V=\operatorname{int} \bar{V}$. With the notations from Proposition 4.1 consider now the problem

$$
\min \left\{\pi_{V}(y) \mid y \in S_{V}, F_{\mu}(y) \geq p\right\}, \quad \text { with } \quad S_{V}=A\left(C_{V}\right)
$$


or, equivalently,

$$
\min \left\{\pi_{V}(y) \mid y \in S_{V}, h(y) \leq 0\right\} \quad \text { where } \quad h(y)=F_{\mu}^{r}(y)-p^{r} .
$$

According to Proposition 4.1 the solution set $Y_{V}(\mu)$ of this problem fulfills $\Psi(\mu)=\Psi_{V}(\mu)=$ $\sigma_{V}\left(Y_{V}(\mu)\right)$. Let $y_{*} \in Y_{V}(\mu)$ and $\bar{y}=A \bar{x}$ with $\bar{x} \in C$ from (iii). Then $r$-concavity of $\mu$ implies for any $\lambda \in(0,1]$ :

$$
\begin{aligned}
h\left(\lambda \bar{y}+(1-\lambda) y_{*}\right) & =F_{\mu}^{r}\left(\lambda \bar{y}+(1-\lambda) y_{*}\right)-p^{r} \leq \lambda F_{\mu}^{r}(\bar{y})+(1-\lambda) F_{\mu}^{r}\left(y_{*}\right)-p^{r} \\
& \leq \lambda\left(F_{\mu}^{r}(\bar{y})-p^{r}\right)<0 .
\end{aligned}
$$

Thus, we may select $\hat{\lambda} \in(0,1]$ such that $\hat{y}=\hat{\lambda} \bar{y}+(1-\hat{\lambda}) y_{*}$ belongs to $S_{V}$ and has the property $h(\hat{y})<0$. This constraint qualification implies the existence of a Kuhn-Tucker coefficient $\lambda_{*} \geq 0$ such that

$$
\pi_{V}\left(y_{*}\right)=\min \left\{\pi_{V}(y)+\lambda_{*} h(y) \mid y \in S_{V}\right\} \quad \text { and } \quad \lambda_{*} h\left(y_{*}\right)=0
$$

In case $\lambda_{*}=0$, this would imply $y_{*} \in \operatorname{argmin}\left\{\pi_{V}(y) \mid y \in S_{V}\right\}$ and, hence, the existence of some $x_{*} \in \Psi(\mu)$ with $g\left(x_{*}\right)=\pi_{V}\left(A x_{*}\right)=\min \left\{g(x) \mid A x=y_{*}, x \in C_{V}\right\}$. Then, in contradiction to condition (ii), $x^{*}$ would minimize $g$ w.r.t. $C$ due to $x^{*} \in$ int $V$. Thus $\lambda_{*}>0$ and $\pi_{V}+\lambda_{*} h$ is strongly convex on $S_{V}$. Hence, $y_{*}$ is the unique minimizer of $\pi_{V}+\lambda_{*} h$ and the growth property

$$
\exists \rho>0 \quad \rho\left\|y-y_{*}\right\|^{2} \leq \pi_{V}(y)+\lambda_{*} h(y)-\pi_{V}\left(y^{*}\right) \quad \forall y \in S_{V}
$$

is valid. From Proposition 4.1 we conclude $\Psi(\mu)=\Psi_{V}(\mu)=\sigma_{V}\left(y_{*}\right)$ and

$$
\left\|A x-y_{*}\right\|^{2} \leq \rho^{-1}\left(\pi_{V}(A x)-\varphi(\mu)\right) \quad \forall x \in C_{V}, F_{\mu}(A x) \geq p .
$$

Now, choose any $x \in C \cap V$ such that $F_{\mu}(A x) \geq p$. Obviously

$$
d(x, \Psi(\mu))=d\left(x, \sigma_{V}\left(y^{*}\right)\right) \leq d\left(x, \sigma_{V}(A x)\right)+d_{H}\left(\sigma_{V}(A x), \sigma_{V}\left(y^{*}\right)\right),
$$

where $d_{H}$ refers to the Hausdorff distance on bounded subsets of $\mathbb{R}^{m}$. Using the last two statements of Proposition 4.1 (with some Hausdorff Lipschitz modulus $L>0$ ) along with (23) we continue by

$$
\begin{aligned}
d(x, \Psi(\mu))^{2} & \leq 2\left(d\left(x, \sigma_{V}(A x)\right)^{2}+d_{H}\left(\sigma_{V}(A x), \sigma_{V}\left(y^{*}\right)\right)^{2}\right) \\
& \leq 2\left(\eta^{-1}\left(g(x)-\pi_{V}(A x)\right)+L^{2}\left\|A x-y_{*}\right\|^{2}\right) \\
& \leq 2\left(\eta^{-1}\left(g(x)-\pi_{V}(A x)\right)+L^{2} \rho^{-1}\left(\pi_{V}(A x)-\varphi(\mu)\right)\right) \\
& \leq 2 \max \left\{\eta^{-1}, L^{2} \rho^{-1}\right\}(g(x)-\varphi(\mu))
\end{aligned}
$$

Together with Theorem 2.1 the preceding result leads to upper Hölder continuity of the localized solution set mapping $\Psi_{V}$ at $\mu$ (with rate $1 / 2$ ) immediately. The class $\mathcal{B}$ of closed subsets of $\mathbb{I R}^{s}$ defining the metric $\alpha$ on $\mathcal{P}\left(\mathbb{R}^{s}\right)$ (see $(3)$ ) specializes to the collection of all left orthants here, i.e., $\mathcal{B}_{K}=\left\{z+\mathbb{R}_{-}^{s} \mid z \in \mathbb{R}^{s}\right\}$. Hence, the metric $\alpha$ becomes the Kolmogorov distance $\alpha_{K}(\mu, \nu)=\sup _{z \in \mathbb{R}^{s}}\left|F_{\mu}(z)-F_{\nu}(z)\right|$. Using the special structure of problem $\mathbf{P}(\mu)$ we are able to show even the Hausdorff Hölder continuity of $\Psi_{V}$ at $\mu$. 
Theorem 4.3 Adopt the setting of Theorem 4.2. Then there exist $L>0, \delta>0$ and a neighbourhood $V$ of $\Psi(\mu)$ with

$$
d_{H}\left(\Psi(\mu), \Psi_{V}(\nu)\right) \leq L\left\|F_{\mu}-F_{\nu}\right\|_{\infty}^{1 / 2} \quad \text { whenever } \quad \nu \in \mathcal{P}\left(\mathbb{R}^{s}\right),\left\|F_{\mu}-F_{\nu}\right\|_{\infty}<\delta .
$$

Here, again, $d_{H}$ denotes the Hausdorff distance and $\left\|F_{\mu}-F_{\nu}\right\|_{\infty}=\sup _{z \in \mathbb{R}^{s}}\left|F_{\mu}(z)-F_{\nu}(z)\right|$.

\section{Proof:}

As in the proof of Theorem 4.2 we construct a polyhedron $\bar{V} \subseteq \mathbb{R}^{m}$ such that $\Psi(\mu)$ is contained in the interior $V$ of $\bar{V}$. Since the assumptions (i) - (iii) of Theorem 2.1 are satisfied (cf. Proposition 2.2) for the metric regularity property, the localized solution-set mapping $\Psi_{V}$ is upper semicontinuous at $\mu$ (w.r.t. the Kolmogorov distance $\alpha_{K}(\mu, \nu)=\left\|F_{\mu}-F_{\nu}\right\|_{\infty}$ ) and $\Psi_{V}(\nu) \neq \emptyset$ is a complete local minimizing set for $\mathbf{P}(\nu)$ if $\alpha_{K}(\mu, \nu)$ is sufficiently small. Hence, there exists a $\delta>0$ such that $\emptyset \neq \Psi_{V}(\nu) \subseteq V$ for all $\nu \in \mathcal{P}\left(\mathbb{R}^{s}\right)$ with $\left\|F_{\mu}-F_{\nu}\right\|_{\infty}<\delta$. With the notations from Proposition 4.1 and using the fact that $Y_{V}(\mu)=\left\{y_{*}\right\}$ and $\Psi(\mu)=$ $\Psi_{V}(\mu)=\sigma_{V}\left(y_{*}\right)$ we obtain

$$
d_{H}\left(\Psi(\mu), \Psi_{V}(\nu)\right)=d_{H}\left(\sigma_{V}\left(y_{*}\right), \sigma_{V}\left(Y_{V}(\nu)\right)\right) \leq \hat{L} \sup _{y \in Y_{V}(\nu)}\left\|y-y_{*}\right\|
$$

where $\hat{L}>0$ is the Hausdorff Lipschitz constant of $\sigma_{V}$ (cf. Proposition 4.1). Using (22), the above chain of inequalities extends to

$$
d_{H}\left(\Psi(\mu), \Psi_{V}(\nu)\right) \leq \hat{L} \rho^{-1 / 2} \sup _{y \in Y_{V}(\nu)}\left[\pi_{V}(y)-\pi_{V}\left(y_{*}\right)\right]^{1 / 2}=\hat{L} \rho^{-1 / 2}\left|\varphi_{V}(\nu)-\varphi_{V}(\mu)\right|^{1 / 2}
$$

Appealing to the Lipschitz property for the localized marginal values (w.r.t. $\alpha_{K}$ ) in Theorem 2.1 completes the proof.

The assumptions (i)-(iv) imposed in the Theorems 4.2 and 4.3 all concern the original problem $\mathbf{P}(\mu)$. Condition (i) is basic for our stability analysis and is satisfied, for example, if $C$ is a polytope. The conditions (ii) and (iii) mean that the probability level $p$ is not chosen too low and too high, respectively. (ii) expresses the fact that the presence of the probabilistic constraint $F_{\mu}(A x) \geq p$ moves the solution set $\Psi(\mu)$ away from that obtained without imposing the reliability constraint for ' $A x \geq \xi$ '. From a modelling point of view, both conditions show the significance of the choice of the reliability level $p$. Assumption (iv) is decisive for the desired growth condition of the objective function around $\Psi(\mu)$. Contrary to the $r$-concavity of $\mu$, which is supposed to hold globally, (iv) requires strong convexity of $F_{\mu}^{r}$ as a local property around $A\left(\Psi(\mu)\right.$ ) (in addition to the convexity of $F_{\mu}^{r}$ on $\mathbb{I R}^{s}$ with values in the extended real numbers). Although no general sufficient criterion for (iv) is available so far, (iv) seems to be satisfied in many cases when $A(\Psi(\mu))$ belongs to the interior of the support of $\mu$. This is illustrated by the following example.

Example 4.4 Let $m=2, s=1, g(x)=x_{1} \forall x=\left(x_{1}, x_{2}\right) \in \mathbb{R}^{2}, A=(1,0), p=1 / 2, C=$ $[-1,1] \times[-1,1]$ and $\mu$ be the uniform distribution on $[-1 / 2,1 / 2]$. Then $\Psi(\mu)=\{0\} \times$ 
$[-1,1], \varphi(\mu)=0$ and for each $r<0$,

$$
F_{\mu}^{r}(z)=\left\{\begin{array}{cll}
\infty & , & z<-1 / 2 \\
(z+1 / 2)^{r} & , & z \in[-1 / 2,1 / 2] \\
1 & , & z>1 / 2
\end{array}\right.
$$

Hence, it holds

$$
\frac{d^{2} F_{\mu}^{r}}{d z^{2}}(z)=r(r-1) F_{\mu}^{r-2}(z)>0
$$

in a neighbourhood of $z=0$, and $F_{\mu}^{r}$ is strongly convex around $A(\Psi(\mu))=\{0\}$. Since the conditions (i)-(iii) are satisfied, both theorems apply. In fact, we have $x_{1}=g\left(x_{1}\right) \geq$ $d(x, \Psi(\mu))^{2}=x_{1}^{2}$ for all $x \in(-1,1) \times(-1,1)$ satisfying $F_{\mu}\left(x_{1}\right) \geq 1 / 2$.

As a conclusion from Theorem 4.3 we finally derive a large deviation result for the Hausdorff distance of solution sets when estimating $\mu$ by empirical measures. As in Section 2 , let $\xi_{1}, \xi_{2}, \ldots, \xi_{n}, \ldots$ be iid $\mathbb{R}^{s}$-valued random variables (on some probability space $(\Omega, \mathcal{A}, P)$ ) having common distribution $\mu$, and let $\mu_{n}=n^{-1} \sum_{i=1}^{n} \delta_{\xi_{i}}$ denote the empirical measure of $\xi_{1}, \ldots, \xi_{n}(n \in \mathbb{N})$. Since $\mathcal{B}_{K}$ is a permissible VC class (cf. Section 2), the proof of our final result follows the same lines as Proposition 2.3 and is therefore omitted.

Corollary 4.5 Adopt the setting of Theorem 4.3. Then it holds for all $\varepsilon>0$,

$$
\limsup _{n \rightarrow \infty} n^{-1} \log P\left(d_{H}\left(\Psi(\mu), \Psi_{V}\left(\mu_{n}\right)\right) \geq \varepsilon\right) \leq-2 \min \left\{\delta^{2}, \varepsilon^{4} L^{-4}\right\},
$$

where $L$ and $\delta$ denote the constants and $V$ the bounded open set arising in Theorem 4.3.

\section{Acknowledgement:}

The authors wish to thank Prof. R.T. Rockafellar (University of Washington, Seattle) and Prof. R.J.-B. Wets (University of California, Davis) for helpful discussions on the subject of this paper and for their hospitality during the authors' visits at Seattle and Davis. Further thanks are due to Darinka Dentcheva (Humboldt-Universität Berlin) and Rüdiger Schultz (Konrad-ZuseZentrum Berlin) for helpful discussions. 


\section{References}

[1] Z. Artstein, "Sensitivity with respect to the underlying information in stochastic programs," Journal of Computational and Applied Mathematics 56 (1994) 127-136.

[2] H. Attouch and R.J.-B. Wets, "Quantitative stability of variational systems II: A framework for nonlinear conditioning," SIAM Journal on Optimization 3 (1993) 359-381.

[3] A. Auslender, "Stability in mathematical programming with nondifferentiable data," SIAM Journal of Control and Optimization 22 (1984) 239-254.

[4] R.N. Bhattacharya and R. Ranga Rao, Normal approximation and asymptotic expansions (Wiley, New York, 1976).

[5] P. Billingsley and F. Topsøe, "Uniformity in weak convergence," Zeitschrift für Wahrscheinlichkeitstheorie und verwandte Gebiete 7 (1967) 1-16.

[6] P. Billingsley, Convergence of probability measures (Wiley, New York, 1968).

[7] J.F. Bonnans and A.D. Ioffe, "Second-order sufficiency and quadratic growth for non isolated minima," INRIA - Report No. 1853(1993) and Mathematics of Operations Research, to appear.

[8] C. Borell, "Convex set functions in d-space," Periodica Mathematica Hungarica 6 (1975) 111-136.

[9] J.M. Borwein, "Stability and regular points of inequality systems," Journal of Optimization Theory and Applications 48 (1986) 9-52.

[10] H.J. Brascamp and E.H. Lieb, "On extensions of the Brunn-Minkowski and PrékopaLeindler theorems, including inequalities for log-concave functions and with an application to the diffusion equation," Journal of Functional Analysis 22 (1976) 366-389.

[11] F.H. Clarke, Optimization and nonsmooth analysis (Wiley, New York, 1983).

[12] S. Das Gupta, "Brunn-Minkowski inequality and its aftermath," Journal of Multivariate Analysis 10 (1980) 296-318.

[13] R.M. Dudley, "A course on empirical processes," Ecole d'Eté de Probabilités de St. Flour 1982, Lecture Notes in Mathematics, vol. 1097, Springer-Verlag, Berlin, 1984, pp. 2-142.

[14] J. Dupačová, "Stability in stochastic programming - probabilistic constraints," in: V.I. Arkin, A. Shiraev and R.J.-B. Wets, eds., Stochastic Optimization, Lecture Notes in Control and Information Sciences vol. 81 (Springer-Verlag, Berlin, 1986) pp. 314-325.

[15] J. Dupačová, "Stability and sensitivity analysis for stochastic programming," Annals of Operations Research 27 (1990) 115-142. 
[16] N. Gröwe, "Numerical solution and asymptotic analysis of stochastic programs," Dissertation, Humboldt University Berlin, 1995.

[17] R. Henrion, "Topological characterization of the approximate subdifferential in the finitedimensional case," ZOR - Mathematical Methods of Operations Research 41 (1995) 161-173.

[18] R. Henrion, "Topological properties of the approximate subdifferential," submitted to Journal of Mathematical Analysis and Applications

[19] A.D. Ioffe, "Approximate subdifferentials and applications. I: The finite-dimensional theory," Transactions of the American Mathematical Society 281 (1984) 389-416.

[20] A. Jourani and L. Thibault, "Approximate subdifferential and metric regularity: The finite dimensional case" Mathematical Programming 47 (1990) 203-218.

[21] A. Jourani, "Constraint qualifications and Lagrange multipliers in nondifferentiable programming problems" Journal of Optimization Theory and Applications 81 (1994) 533-548.

[22] P. Kall, "On approximations and stability in stochastic programming," in: J. Guddat, H.Th. Jongen, B. Kummer and F. Nožička, eds., Parametric optimization and related topics (Akademie-Verlag, Berlin, 1987) pp. 387-407.

[23] V. Kan̆ková, "On the convergence rate of empirical estimates in chance constrained stochastic programming," Kybernetika 26 (1990) 449-461.

[24] V. Kan̆ková, "A note on estimates in stochastic programming," Journal of Computational and Applied Mathematics 56 (1994) 97-112.

[25] D. Klatte, "A note on quantitative stability results in nonlinear optimization," in: K. Lommatzsch, ed., Proceedings of the 19. Jahrestagung Mathematische Optimierung (Seminarbericht No. 90, Humboldt University Berlin, Department of Mathematics, 1987) pp. $77-86$.

[26] D. Klatte, "On quantitative stability for non-isolated minima," Control and Cybernetics 23 (1994) 183-200.

[27] D. Klatte and G. Thiere, "Error bounds for solutions of linear equations and inequalities," ZOR - Mathematical Methods of Operations Research 41 (1995) 191-214.

[28] B. Kummer, "Linearly and nonlinearly perturbed optimization problems in finite dimension," in: J. Guddat, H.Th. Jongen, B. Kummer and F. Nožička eds., Parametric Optimization and Related Topics (Akademie-Verlag, Berlin, 1987) pp. 249-267.

[29] R. Lucchetti, G. Salinetti and R.J.-B. Wets, "Uniform convergence of probability measures: Topological criteria," Journal of Multivariate Analysis 51 (1994) 252-264.

[30] K. Marti, "Stochastic optimization in structural design," Zeitschrift für Angewandte Mathematik und Mechanik 72 (1992) T452-T464. 
[31] J. Mayer, "Computational techniques for probabilistic constrained optimization problems," in: K. Marti, ed., Stochastic Optimization, Lecture Notes in Economics and Mathematical Systems, vol. 379, Springer-Verlag, Berlin, 1992, pp.141-164.

[32] B.S. Mordukhovich, "Complete characterization of openness, metric regularity and Lipschitzian properties of multifunctions," Transactions of the American Mathematical Society 340 (1993) 1-35.

[33] B.S. Mordukhovich, "Generalized differential calculus for nonsmooth and set-valued mappings," Journal of Mathematical Analysis and Applications 183 (1994) 250-288.

[34] V.I. Norkin, "The analysis and optimization of probability functions," IIASA working paper WP-93-6, International Institute for Applied Systems Analysis, Laxenburg (Austria), 1993.

[35] J.P. Penot, "Metric regularity, openness and Lipschitzian behavior of multifunctions," Nonlinear Analysis Theory, Methods and Applications 13 (1989) 629-643.

[36] D. Pollard, Convergence of stochastic processes (Springer-Verlag, New York, 1984).

[37] A. Prékopa, "Logarithmic concave measures with applications to stochastic programming," Acta Scientiarum Mathematicarum 32 (1971) 301-316.

[38] A. Prékopa, Stochastic Programming (Kluwer Academic Publishers, Dordrecht, 1995).

[39] S.M. Robinson, "Local epi-continuity and local optimization," Mathematical Programming 37 (1987) 208-223.

[40] R.T. Rockafellar, "Lipschitzian properties of multifunctions," Nonlin. Anal. Th. Math. Appl. 9 (1985) 867-885.

[41] R.T. Rockafellar, "Integral functionals, normal integrands and measurable selections," in: G.P. Gossez et al. eds., Nonlinear Operators and the Calculus of Variations, Lecture Notes in Mathematics, vol. 543, Springer-Verlag, New York, 1976, pp. 157-207.

[42] W. Römisch and R. Schultz, "Distribution sensitivity in stochastic programming," Mathematical Programming 50 (1991) 197-226.

[43] W. Römisch and R. Schultz, "Distribution sensitivity for certain classes of chanceconstrained models with application to power dispatch," Journal of Optimization Theory and Applications 71 (1991) 569-588.

[44] W. Römisch and R. Schultz, "Stability analysis for stochastic programs," Annals of Operations Research 30 (1991) 241-266.

[45] W. Römisch and R. Schultz, "Lipschitz stability for stochastic programs with complete recourse," SIAM Journal on Optimization 1996, to appear. 
[46] W. Römisch and A. Wakolbinger, "Obtaining convergence rates for approximations in stochastic programming," in: J. Guddat, H.Th. Jongen, B. Kummer and F. Nožička, eds., Parametric optimization and related topics (Akademie-Verlag, Berlin, 1987) pp. 327-343.

[47] G. Salinetti, "Approximations for chance-constrained programming problems," Stochastics 10 (1983) 157-179.

[48] A. Shapiro, "Asymptotic analysis of stochastic programs," Annals of Operations Research 30 (1991) 169-186.

[49] A. Shapiro, "Perturbation analysis of optimization problems in Banach spaces," Numerical Functional Analysis and Optimization 13 (1992) 97-116.

[50] G.R. Shorack and J.A. Wellner, Empirical processes with applications to statistics (Wiley, New York, 1986).

[51] S. Vogel, "Stability results for stochastic programming problems", Optimization 19 (1988) 269-288.

[52] S. Vogel, "On stability in multiobjective programming - a stochastic approach," Mathematical Programming 56 (1992) 91-119.

[53] J. Wang, "Continuity of feasible solution sets of probabilistic constrained programs," Journal of Optimization Theory and Applications 63 (1989) 79-89.

[54] R.J.-B. Wets, "Stochastic programming," in: G.L. Nemhauser, A.H.G. Rinnoy Kan and M.J. Todd eds., Handbooks in operations research and management science. Volume 1: Optimization (North-Holland, 1989) pp. 573-629.

[55] R.J.-B. Wets, "Challenges in Stochastic Programming," IIASA working paper WP-94-32, International Institute for Applied Systems Analysis, Laxenburg (Austria), 1994. 